Volume 25 (2019) 12-34

DOI: $10.24330 /$ ieja. 504101

\title{
FREE MODIFIED ROTA-BAXTER ALGEBRAS AND HOPF ALGEBRAS
}

\author{
Xigou Zhang, Xing Gao and Li Guo \\ Received: 16 January 2018; Revised: 28 July 2018; Accepted: 7 September 2018 \\ Communicated by Christian Lomp
}

\begin{abstract}
The notion of a modified Rota-Baxter algebra comes from the combination of those of a Rota-Baxter algebra and a modified Yang-Baxter equation. In this paper, we first construct free modified Rota-Baxter algebras. We then equip a free modified Rota-Baxter algebra with a bialgebra structure by a cocycle construction. Under the assumption that the generating algebra is a connected bialgebra, we further equip the free modified Rota-Baxter algebra with a Hopf algebra structure.
\end{abstract}

Mathematics Subject Classification (2010): 16T99, 16W99, 16S10

Keywords: Modified Rota-Baxter algebra, Rota-Baxter algebra, Hopf algebra, bracketed word, cocycle

\section{Introduction}

This paper studies free objects in the category of modified Rota-Baxter algebras, a concept coming from the combination of a Rota-Baxter algebra and a modified Yang-Baxter equation. It also equips the free objects with bialgebra and Hopf algebra structures.

For a fixed constant $\lambda$, a Rota-Baxter operator of weight $\lambda$ is a linear operator $P$ on an associative algebra $R$ that satisfies the Rota-Baxter equation:

$$
P(x) P(y)=P(P(x) y)+P(x P(y))+\lambda P(x y), \quad \forall x, y \in R .
$$

An associative algebra $R$ equipped with a Rota-Baxter operator is called a RotaBaxter algebra, a notion originated from the probability study of G. Baxter [8] in 1960. Later it attracted the attention of well-known mathematicians such as Atkinson, Cartier and Rota $[2,10,26]$. After some years of dormancy, its study experienced a quite remarkable renascence since late 1990s, with many applications

This work was supported by the National Natural Science Foundation of China (No. 11771190), the Fundamental Research Funds for the Central Universities (No. lzujbky-2017-162) and the Natural Science Foundation of Gansu Province (No. 17JR5RA175). 
in mathematics and physics $[1,3,14,17,19,21,22,24,25]$. In particular, it appeared as one of the fundamental algebraic structures in the profound work of Connes and Kreimer on renormalization of quantum field theory [11]. See [16] for further details and references.

The concept of the classical Yang-Baxter equation arose from the study of inverse scattering theory and is also related to Schouten bracket in differential geometry. Further it can be regarded as the classical limit of the quantum Yang-Baxter equation, named after C. N. Yang and R. Baxter. In the 1980s, Semonov-TianShansky [27] found that, under suitable conditions, the operator form of the classical Yang-Baxter equation is precisely the Rota-Baxter identity (1) (of weight 0) on a Lie algebra. As a modified form of the operator form of the classical Yang-Baxter equation, he also introduced in that paper the modified classical Yang-Baxter equation:

$$
[P(x), P(y)]=P[P(x), y]+P[x, P(y)]-[x, y],
$$

later found applications in the study of generalized Lax pairs and affine geometry on Lie groups $[4,9,20]$. As the associative analogue of Eq. (2), the equation

$$
P(x) P(y)=P(P(x) y)+P(x P(y))-x y .
$$

is called the modified associative Yang-Baxter equation, which has been applied to the study of extended $\mathcal{O}$-operators, associative Yang-Baxter equations, infinitesimal bialgebras and dendriform algebras $[6,7,12]$.

In the spirit of the aforementioned Yang-Baxter equation to Rota-Baxter operator connection, a linear operator $P$ satisfying Eq. (3) is called a modified RotaBaxter operator and an associative algebra $R$ equipped with a modified Rota-Baxter operator is called a modified Rota-Baxter algebra.

Integrating the notions of the Rota-Baxter algebra and modified Rota-Baxter algebra, the concept of a modified Rota-Baxter algebra with a weight was introduced in [6] as a special case of extended $\mathcal{O}$-operators in connection with the extended associative Yang-Baxter equation. The latter motivated their study in the Lie algebra context [5]. In [29], free commutative modified Rota-Baxter algebras were constructed by means of a modified quasi-shuffle product and modified stuffle product, in analogy to the case of free commutative Rota-Baxter algebras [10,17].

Considering the close relationship between the modified Rota-Baxter (associative) algebras and the modified Yang-Baxter equation for Lie algebras, it is especially interesting to consider noncommutative modified Rota-Baxter algebras. This is the subject of study of this paper, focusing on the construction of the free objects 
and the Hopf algebra structures on the free objects. More precisely, in Section 2, we obtain an explicit construction of the free modified Rota-Baxter algebra on an algebra, by giving a natural basis of the algebra and the corresponding multiplication table. In Section 3, we further provide a bialgebra and then a Hopf algebra structure on the free modified Rota-Baxter algebra.

Notations. For the rest of this paper, unless otherwise specified, algebras are associative unitary algebras over a commutative unitary ring $\mathbf{k}$.

\section{Free modified Rota-Baxter algebras}

In this section we construct free modified Rota-Baxter algebras. We give the construction in Section 2.1, leading to the main Theorem 2.6 of this section. The proof of the theorem is completed in Section 2.2.

\subsection{The general construction of the free modified Rota-Baxter algebras.}

We begin with the general definition of modified Rota-Baxter algebras.

Definition 2.1. Let $R$ be a k-algebra and $\kappa \in \mathbf{k}$. A linear map $P: R \rightarrow R$ is called a modified Rota-Baxter operator of weight $\kappa$ if $P$ satisfies the operator identity

$$
P(u) P(v)=P(u P(v))+P(P(u) v)+\kappa u v, \quad \text { for all } u, v \in R .
$$

Then the pair $(R, P)$ or simply $R$ is called a modified Rota-Baxter algebra of weight $\kappa$.

Together with the algebra homomorphisms between the algebras that preserves the linear operators, the class of modified Rota-Baxter algebras of weight $\kappa$ forms a category. We refer the reader to [29] and the references therein for basic properties of modified Rota-Baxter algebras and focus our attention to the construction of free modified Rota-Baxter algebras. We first give the definition.

Definition 2.2. Let $A$ be a k-algebra. A free modified Rota-Baxter algebra on $A$ is a modified Rota-Baxter algebra $\left(F(A), P_{A}\right)$ together with an algebra homomorphism $j: A \longrightarrow F(A)$ with the property that, for any given modified Rota-Baxter algebra $(R, P)$ and algebra homomorphism $f: A \longrightarrow R$, there is a unique homomorphism $\bar{f}: F(A) \longrightarrow R$ of modified Rota-Baxter algebras such that $\bar{f} j=f$.

Note that taking $A$ to be the free algebra $\mathbf{k}\langle Y\rangle$ on a set $Y$, we obtain the free modified Rota-Baxter algebra on the set $Y$. Let $A$ be a k-algebra with a k-basis $X$. We first display a $\mathbf{k}$-basis $\mathfrak{X}_{\infty}$ of free modified Rota-Baxter algebras in terms of bracketed words from the alphabet set $X$. 
Remark 2.3. The set $\mathfrak{X}_{\infty}$ is called the set of Rota-Baxter words that was applied to construct free Rota-Baxter algebras [14]. Enumeration properties and generating functions of Rota-Baxter words were obtained in [18] to which we refer the reader for further details.

Let $\lfloor$ and $\rfloor$ be two different symbols not in $X$, called brackets, and let $X^{\prime}:=$ $X \cup\{\lfloor\rfloor$,$\} . Denote by M\left(X^{\prime}\right)$ the free monoid generated by $X^{\prime}$.

Definition 2.4. ([13,16]) Let $Y, Z$ be two subsets of $M\left(X^{\prime}\right)$. Define the alternating product of $Y$ and $Z$ to be

$\Lambda(Y, Z)=\left(\bigsqcup_{r \geq 1}(Y\lfloor Z\rfloor)^{r}\right) \bigsqcup\left(\bigsqcup_{r \geq 0}(Y\lfloor Z\rfloor)^{r} Y\right) \bigsqcup\left(\bigsqcup_{r \geq 1}(\lfloor Z\rfloor Y)^{r}\right) \bigsqcup\left(\bigsqcup_{r \geq 0}(\lfloor Z\rfloor Y)^{r}\lfloor Z\rfloor\right)$.

Here $\sqcup$ stands for disjoint union.

For example, $y_{1}\left\lfloor z_{1}\right\rfloor y_{2},\left\lfloor z_{1}\right\rfloor y_{1}\left\lfloor z_{2}\right\rfloor, y_{1}, y_{2} \in Y, z_{1}, z_{2} \in Z$, are elements in $\Lambda(Y, Z)$. But $\left\lfloor z_{1}\right\rfloor\left\lfloor z_{2}\right\rfloor$ are not in $\Lambda(Y, Z)$.

We construct a sequence $\mathfrak{X}_{n}$ of subsets of $M\left(X^{\prime}\right)$ by the following recursion on $n \geq 0$. For the initial step, we define $\mathfrak{X}_{0}:=X \cup\{1\}$. For the inductive step, we define

$$
\mathfrak{X}_{n+1}:=\Lambda\left(X, \mathfrak{X}_{n}\right) \cup\{1\} \text { for } n \geq 1 .
$$

For example, for $x_{1}, x_{2}, x_{3} \in X$, the elements $\left\lfloor x_{1}\right\rfloor x_{2}\left\lfloor x_{3}\right\rfloor, x_{1}\left\lfloor x_{2}\left\lfloor x_{3}\right\rfloor\right\rfloor$ and $\left\lfloor\left\lfloor x_{1}\right\rfloor x_{2}\left\lfloor\left\lfloor x_{3}\right\rfloor\right\rfloor\right\rfloor$ are all in $\mathfrak{X}_{3}$, the first two are in $\mathfrak{X}_{2}$ and the first one is in $\mathfrak{X}_{1}$.

From the definition we have $\mathfrak{X}_{1} \supseteq \mathfrak{X}_{0}$. Assuming $\mathfrak{X}_{n} \supseteq \mathfrak{X}_{n-1}$, we get

$$
\mathfrak{X}_{n+1}=\Lambda\left(X, \mathfrak{X}_{n}\right) \supseteq \Lambda\left(X, \mathfrak{X}_{n-1}\right)=\mathfrak{X}_{n} .
$$

Thus we can define

$$
\mathfrak{X}_{\infty}:=\lim _{\longrightarrow} \mathfrak{X}_{n}=\bigcup_{n \geq 0} \mathfrak{X}_{n} .
$$

For $\mathfrak{x} \in \mathfrak{X}_{\infty}$, we define the depth $\operatorname{dep}(\mathfrak{x})$ of $\mathfrak{x}$ to be

$$
\operatorname{dep}(\mathfrak{x}):=\min \left\{n \mid \mathfrak{x} \in \mathfrak{X}_{n}\right\} .
$$

Further, every $\mathfrak{x} \in \mathfrak{X}_{\infty} \backslash\{1\}$ has a unique standard decomposition:

$$
\mathfrak{x}=\mathfrak{x}_{1} \cdots \mathfrak{x}_{b},
$$

where $\mathfrak{x}_{i}, 1 \leq i \leq b$, are alternatively in $X$ or in $\left\lfloor\mathfrak{X}_{\infty}\right\rfloor$. We call $b$ to be the breadth of $\mathfrak{x}$, denoted by bre( $\mathfrak{x}$ ). We define the head $h(\mathfrak{x})$ of $\mathfrak{x}$ to be 0 (resp. 1) if $\mathfrak{x}_{1}$ is in $X$ (resp. in $\left\lfloor\mathfrak{X}_{\infty}\right\rfloor$ ). Similarly define the tail $t(\mathfrak{x})$ of $\mathfrak{x}$ to be 0 (resp. 1) if $\mathfrak{x}_{b}$ is in $X$ (resp. in $\left\lfloor\mathfrak{X}_{\infty}\right\rfloor$ ). 
Fix a $\kappa \in \mathbf{k}$. We will equip the free $\mathbf{k}$-module

$$
F_{\kappa}(A)=\mathbf{k} \mathfrak{X}_{\infty}=\bigoplus_{\mathfrak{x} \in \mathfrak{X}_{\infty}} \mathbf{k x}
$$

with a multiplication $\diamond:=\diamond_{\kappa}$. This is accomplished by defining $\mathfrak{x} \diamond \mathfrak{x}^{\prime} \in F_{\kappa}(A)$ for basis elements $\mathfrak{x}, \mathfrak{x}^{\prime} \in \mathfrak{X}_{\infty}$ and then extending bilinearly. Roughly speaking, the product of $\mathfrak{x}$ and $\mathfrak{x}^{\prime}$ is defined to be the concatenation whenever $t(\mathfrak{x}) \neq h\left(\mathfrak{x}^{\prime}\right)$. When $t(\mathfrak{x})=h\left(\mathfrak{x}^{\prime}\right)$, the product is defined by the product in $A$ or by the modified Rota-Baxter identity in Eq. (4).

To be precise, we use induction on the sum $n:=\operatorname{dep}(\mathfrak{x})+\operatorname{dep}\left(\mathfrak{x}^{\prime}\right) \geq 0$ to define $\mathfrak{x} \diamond \mathfrak{x}^{\prime}$. For the initial step of $n=0, \mathfrak{x}, \mathfrak{x}^{\prime}$ are in $X$ and so are in $A$. Then we define

$$
\mathfrak{x} \diamond \mathfrak{x}^{\prime}:=\mathfrak{x} \cdot \mathfrak{x}^{\prime} \in A \subseteq F_{\kappa}(A) .
$$

Here $\cdot$ is the product in $A$.

For the inductive step, let $k \geq 0$ be given and assume that $\mathfrak{x} \diamond \mathfrak{x}^{\prime}$ have been defined for all $\mathfrak{x}, \mathfrak{x}^{\prime} \in \mathfrak{X}_{\infty}$ with $n=\operatorname{dep}(\mathfrak{x})+\operatorname{dep}\left(\mathfrak{x}^{\prime}\right) \leq k$. Then consider $\mathfrak{x}, \mathfrak{x}^{\prime} \in \mathfrak{X}_{\infty}$ with $n=\operatorname{dep}(\mathfrak{x})+\operatorname{dep}\left(\mathfrak{x}^{\prime}\right)=k+1$. First treat the case when bre $(\mathfrak{x})=\operatorname{bre}\left(\mathfrak{x}^{\prime}\right)=1$. Then $\mathfrak{x}$ and $\mathfrak{x}^{\prime}$ are in $X$ or $\left\lfloor\mathfrak{X}_{\infty}\right\rfloor$. Since $n=k+1 \geq 1, \mathfrak{x}$ and $\mathfrak{x}^{\prime}$ cannot be both in $X$. We accordingly define

$$
\mathfrak{x} \diamond \mathfrak{x}^{\prime}:= \begin{cases}\mathfrak{x x}^{\prime}, & \text { if } \mathfrak{x} \in X \text { and } \mathfrak{x}^{\prime} \in\left\lfloor\mathfrak{X}_{\infty}\right\rfloor, \\ \mathfrak{x} \mathfrak{x}^{\prime}, & \text { if } \mathfrak{x} \in\left\lfloor\mathfrak{X}_{\infty}\right\rfloor \text { and } \mathfrak{x}^{\prime} \in X, \\ \left\lfloor\lfloor\overline{\mathfrak{x}}\rfloor \diamond \overline{\mathfrak{x}}^{\prime}\right\rfloor+\left\lfloor\overline{\mathfrak{x}} \diamond\left\lfloor\overline{\mathfrak{x}}^{\prime}\right\rfloor\right\rfloor+\kappa \overline{\mathfrak{x}} \diamond \overline{\mathfrak{x}}^{\prime}, & \text { if } \mathfrak{x}=\lfloor\overline{\mathfrak{x}}\rfloor \text { and } \mathfrak{x}^{\prime}=\left\lfloor\overline{\mathfrak{x}}^{\prime}\right\rfloor \in\left\lfloor\mathfrak{X}{ }_{\infty}\right\rfloor .\end{cases}
$$

Here the product in the first and second case are by concatenation and in the third case is by the induction hypothesis since for the three products on the right hand side we have

$$
\begin{aligned}
\operatorname{dep}(\lfloor\overline{\mathfrak{x}}\rfloor)+\operatorname{dep}\left(\overline{\mathfrak{x}}^{\prime}\right) & =\operatorname{dep}(\lfloor\overline{\mathfrak{x}}\rfloor)+\operatorname{dep}\left(\left\lfloor\overline{\mathfrak{x}}^{\prime}\right\rfloor\right)-1=\operatorname{dep}(\mathfrak{x})+\operatorname{dep}\left(\mathfrak{x}^{\prime}\right)-1=k, \\
\operatorname{dep}(\overline{\mathfrak{x}})+\operatorname{dep}\left(\left\lfloor\overline{\mathfrak{x}}^{\prime}\right\rfloor\right) & =\operatorname{dep}(\lfloor\overline{\mathfrak{x}}\rfloor)+\operatorname{dep}\left(\left\lfloor\overline{\mathfrak{x}}^{\prime}\right\rfloor\right)-1=\operatorname{dep}(\mathfrak{x})+\operatorname{dep}\left(\mathfrak{x}^{\prime}\right)-1=k, \\
\operatorname{dep}(\overline{\mathfrak{x}})+\operatorname{dep}\left(\overline{\mathfrak{x}}^{\prime}\right) & =\operatorname{dep}(\lfloor\overline{\mathfrak{x}}\rfloor)-1+\operatorname{dep}\left(\left\lfloor\overline{\mathfrak{x}}^{\prime}\right\rfloor\right)-1=\operatorname{dep}(\mathfrak{x})+\operatorname{dep}\left(\mathfrak{x}^{\prime}\right)-2=k-1 .
\end{aligned}
$$

We next treat the case when bre $(\mathfrak{x})>1$ or bre $\left(\mathfrak{x}^{\prime}\right)>1$. Let $\mathfrak{x}=\mathfrak{x}_{1} \cdots \mathfrak{x}_{b}$ and $\mathfrak{x}^{\prime}=\mathfrak{x}_{1}^{\prime} \cdots \mathfrak{x}_{b^{\prime}}^{\prime}$ be the standard decompositions from Eq. (5). We then define

$$
\mathfrak{x} \diamond \mathfrak{x}^{\prime}=\mathfrak{x}_{1} \cdots \mathfrak{x}_{b-1}\left(\mathfrak{x}_{b} \diamond \mathfrak{x}_{1}^{\prime}\right) \mathfrak{x}_{2}^{\prime} \cdots \mathfrak{x}_{b^{\prime}}^{\prime}
$$

where $\mathfrak{x}_{b} \diamond \mathfrak{x}_{1}^{\prime}$ is defined by Eq. (7) and the rest is given by concatenation. Extending $\diamond$ bilinearly, we obtain a binary operation

$$
\diamond: F_{\kappa}(A) \otimes F_{\kappa}(A) \rightarrow F_{\kappa}(A) .
$$


This completes the definition of $\diamond$.

Lemma 2.5. Let $\mathfrak{x}, \mathfrak{x}^{\prime} \in \mathfrak{X}_{\infty}$.

(a) $h(\mathfrak{x})=h\left(\mathfrak{x} \diamond \mathfrak{x}^{\prime}\right)$ and $t\left(\mathfrak{x}^{\prime}\right)=t\left(\mathfrak{x} \diamond \mathfrak{x}^{\prime}\right)$.

(b) If $t(\mathfrak{x}) \neq h\left(\mathfrak{x}^{\prime}\right)$, then $\mathfrak{x} \diamond \mathfrak{x}^{\prime}=\mathfrak{x x}^{\prime}$ (concatenation).

(c) If $t(\mathfrak{x}) \neq h\left(\mathfrak{x}^{\prime}\right)$, then for any $\mathfrak{x}^{\prime \prime} \in \mathfrak{X}_{\infty}$,

$$
\left(\mathfrak{x} \mathfrak{x}^{\prime}\right) \diamond \mathfrak{x}^{\prime \prime}=\mathfrak{x}\left(\mathfrak{x}^{\prime} \diamond \mathfrak{x}^{\prime \prime}\right) \text { and } \mathfrak{x}^{\prime \prime} \diamond\left(\mathfrak{x x}^{\prime}\right)=\left(\mathfrak{x}^{\prime \prime} \diamond \mathfrak{x}\right) \mathfrak{x}^{\prime} .
$$

Proof. Items (a) and (b) follow from the definition of $\diamond$. The proof of Item (c) is the same as [16, Lemma 4.4.5].

We next define a linear operator

$$
P_{A}: F_{\kappa}(A) \rightarrow F_{\kappa}(A), \mathfrak{x} \mapsto\lfloor\mathfrak{x}\rfloor .
$$

In the rest of the paper, we will use the infix notation $\lfloor\mathfrak{x}\rfloor$ interchangeably with $P_{A}(\mathfrak{x})$ for any $\mathfrak{x} \in F_{\kappa}(A)$. Let

$$
j_{X}: X \hookrightarrow \mathfrak{X}_{\infty} \hookrightarrow F_{\kappa}(A)
$$

be the natural injection which extends to an algebra injection

$$
j_{A}: A \rightarrow F_{\kappa}(A) .
$$

Now we state our first main result, to be proved in the next subsection.

Theorem 2.6. Let $A$ be a $\mathbf{k}$-algebra with a $\mathbf{k}$-basis $X$ and $\kappa \in \mathbf{k}$ be given.

(a) The pair $\left(F_{\kappa}(A), \diamond\right)$ is an algebra.

(b) The triple $\left(F_{\kappa}(A), \diamond, P_{A}\right)$ is a modified Rota-Baxter algebra of weight $\kappa$.

(c) The triple $\left(F_{\kappa}(A), \diamond, P_{A}\right)$ together with the embedding $j_{A}$ is the free modified Rota-Baxter algebra of weight $\kappa$ on the algebra $A$.

\subsection{The proof of Theorem 2.6.}

Proof. (a) It is enough to verify the associativity for basis elements:

$$
\left(\mathfrak{x}^{\prime} \diamond \mathfrak{x}^{\prime \prime}\right) \diamond \mathfrak{x}^{\prime \prime \prime}=\mathfrak{x}^{\prime} \diamond\left(\mathfrak{x}^{\prime \prime} \diamond \mathfrak{x}^{\prime \prime \prime}\right), \text { for all } \mathfrak{x}^{\prime}, \mathfrak{x}^{\prime \prime}, \mathfrak{x}^{\prime \prime \prime} \in \mathfrak{X}_{\infty} .
$$

We carry out the verification by induction on the sum of the depths

$$
n:=\operatorname{dep}\left(\mathfrak{x}^{\prime}\right)+\operatorname{dep}\left(\mathfrak{x}^{\prime \prime}\right)+\operatorname{dep}\left(\mathfrak{x}^{\prime \prime \prime}\right) \geq 0 .
$$


If $n=0$, then

$$
\operatorname{dep}\left(\mathfrak{x}^{\prime}\right)=\operatorname{dep}\left(\mathfrak{x}^{\prime \prime}\right)=\operatorname{dep}\left(\mathfrak{x}^{\prime \prime \prime}\right)=0
$$

and so $\mathfrak{x}^{\prime}, \mathfrak{x}^{\prime \prime}, \mathfrak{x}^{\prime \prime \prime} \in X$. In this case the product $\diamond$ is given by the product in $A$ and so is associative.

Assume that Eq. (9) holds for $n \leq k$ for any given $k \geq 0$ and consider $\mathfrak{x}^{\prime}, \mathfrak{x}^{\prime \prime}, \mathfrak{x}^{\prime \prime \prime} \in$ $\mathfrak{X}_{\infty}$ with

$$
n=\operatorname{dep}\left(\mathfrak{x}^{\prime}\right)+\operatorname{dep}\left(\mathfrak{x}^{\prime \prime}\right)+\operatorname{dep}\left(\mathfrak{x}^{\prime \prime \prime}\right)=k+1 \geq 1 .
$$

If $t\left(\mathfrak{x}^{\prime}\right) \neq h\left(\mathfrak{x}^{\prime \prime}\right)$, then by Lemma 2.5 ,

$$
\left(\mathfrak{x}^{\prime} \diamond \mathfrak{x}^{\prime \prime}\right) \diamond \mathfrak{x}^{\prime \prime \prime}=\left(\mathfrak{x}^{\prime} \mathfrak{x}^{\prime \prime}\right) \diamond \mathfrak{x}^{\prime \prime \prime}=\mathfrak{x}^{\prime}\left(\mathfrak{x}^{\prime \prime} \diamond \mathfrak{x}^{\prime \prime \prime}\right)=\mathfrak{x}^{\prime} \diamond\left(\mathfrak{x}^{\prime \prime} \diamond \mathfrak{x}^{\prime \prime \prime}\right) .
$$

A similar argument holds when $t\left(\mathfrak{x}^{\prime \prime}\right) \neq h\left(\mathfrak{x}^{\prime \prime \prime}\right)$. Thus we only need to verify the associativity when $t\left(\mathfrak{x}^{\prime}\right)=h\left(\mathfrak{x}^{\prime \prime}\right)$ and $t\left(\mathfrak{x}^{\prime \prime}\right)=h\left(\mathfrak{x}^{\prime \prime \prime}\right)$. We next reduce the proof to the breadths of the words and depart to show a lemma.

Lemma 2.7. If Eq. (9) holds for all $\mathfrak{x}^{\prime}, \mathfrak{x}^{\prime \prime}$ and $\mathfrak{x}^{\prime \prime \prime}$ in $\mathfrak{X}_{\infty}$ of breadth one, then it holds for all $\mathfrak{x}^{\prime}, \mathfrak{x}^{\prime \prime}$ and $\mathfrak{x}^{\prime \prime \prime}$ in $\mathfrak{X}_{\infty}$.

Proof. We use induction on the sum of breadths $m:=\operatorname{bre}\left(\mathfrak{x}^{\prime}\right)+\operatorname{bre}\left(\mathfrak{x}^{\prime \prime}\right)+\operatorname{bre}\left(\mathfrak{x}^{\prime \prime \prime}\right) \geq$ 3. The case when $m=3$ is the assumption of the lemma. Assume the associativity holds for $m \leq j$ for some $j \geq 3$ and take $\mathfrak{x}^{\prime}, \mathfrak{x}^{\prime \prime}, \mathfrak{x}^{\prime \prime \prime} \in \mathfrak{X}_{\infty}$ with $m=j+1 \geq 4$. So at least one of $\mathfrak{x}^{\prime}, \mathfrak{x}^{\prime \prime}, \mathfrak{x}^{\prime \prime \prime}$ has breadth greater than or equal to 2 .

First assume that $\operatorname{bre}\left(\mathfrak{x}^{\prime}\right) \geq 2$. Then we may write

$$
\mathfrak{x}^{\prime}=\mathfrak{x}_{1}^{\prime} \mathfrak{x}_{2}^{\prime}, \text { where } \mathfrak{x}_{1}^{\prime}, \mathfrak{x}_{2}^{\prime} \in \mathfrak{X}_{\infty} \text { and } t\left(\mathfrak{x}_{1}^{\prime}\right) \neq h\left(\mathfrak{x}_{2}^{\prime}\right)
$$

By Lemma 2.5, we obtain

$$
\left(\mathfrak{x}^{\prime} \diamond \mathfrak{x}^{\prime \prime}\right) \diamond \mathfrak{x}^{\prime \prime \prime}=\left(\left(\mathfrak{x}_{1}^{\prime} \mathfrak{x}_{2}^{\prime}\right) \diamond \mathfrak{x}^{\prime \prime}\right) \diamond \mathfrak{x}^{\prime \prime \prime}=\left(\mathfrak{x}_{1}^{\prime}\left(\mathfrak{x}_{2}^{\prime} \diamond \mathfrak{x}^{\prime \prime}\right)\right) \diamond \mathfrak{x}^{\prime \prime \prime}=\mathfrak{x}_{1}^{\prime}\left(\left(\mathfrak{x}_{2}^{\prime} \diamond \mathfrak{x}^{\prime \prime}\right) \diamond \mathfrak{x}^{\prime \prime \prime}\right) .
$$

Similarly,

$$
\mathfrak{x}^{\prime} \diamond\left(\mathfrak{x}^{\prime \prime} \diamond \mathfrak{x}^{\prime \prime \prime}\right)=\left(\mathfrak{x}_{1}^{\prime} \mathfrak{x}_{2}^{\prime}\right) \diamond\left(\mathfrak{x}^{\prime \prime} \diamond \mathfrak{x}^{\prime \prime \prime}\right)=\mathfrak{x}_{1}^{\prime}\left(\mathfrak{x}_{2}^{\prime} \diamond\left(\mathfrak{x}^{\prime \prime} \diamond \mathfrak{x}^{\prime \prime \prime}\right)\right)
$$

Thus

$$
\left(\mathfrak{x}^{\prime} \diamond \mathfrak{x}^{\prime \prime}\right) \diamond \mathfrak{x}^{\prime \prime \prime}=\mathfrak{x}^{\prime} \diamond\left(\mathfrak{x}^{\prime \prime} \diamond \mathfrak{x}^{\prime \prime \prime}\right)
$$

whenever

$$
\left(\mathfrak{x}_{2}^{\prime} \diamond \mathfrak{x}^{\prime \prime}\right) \diamond \mathfrak{x}^{\prime \prime \prime}=\mathfrak{x}_{2}^{\prime} \diamond\left(\mathfrak{x}^{\prime \prime} \diamond \mathfrak{x}^{\prime \prime \prime}\right),
$$

which follows from the induction hypothesis. A similar proof works if bre $\left(\mathfrak{x}^{\prime \prime \prime}\right) \geq 2$.

Finally if bre $\left(\mathfrak{x}^{\prime \prime}\right) \geq 2$, we may write

$$
\mathfrak{x}^{\prime \prime}=\mathfrak{x}_{1}^{\prime \prime} \mathfrak{x}_{2}^{\prime \prime} \text { where } \mathfrak{x}_{1}^{\prime \prime}, \mathfrak{x}_{2}^{\prime \prime} \in \mathfrak{X}_{\infty} \text { and } t\left(\mathfrak{x}_{1}^{\prime \prime}\right) \neq h\left(\mathfrak{x}_{2}^{\prime \prime}\right) .
$$


By Lemma 2.5 again, we get

$$
\left(\mathfrak{x}^{\prime} \diamond \mathfrak{x}^{\prime \prime}\right) \diamond \mathfrak{x}^{\prime \prime \prime}=\left(\mathfrak{x}^{\prime} \diamond\left(\mathfrak{x}_{1}^{\prime \prime} \mathfrak{x}_{2}^{\prime \prime}\right)\right) \diamond \mathfrak{x}^{\prime \prime \prime}=\left(\left(\mathfrak{x}^{\prime} \diamond \mathfrak{x}_{1}^{\prime \prime}\right) \mathfrak{x}_{2}^{\prime \prime}\right) \diamond \mathfrak{x}^{\prime \prime \prime}=\left(\mathfrak{x}^{\prime} \diamond \mathfrak{x}_{1}^{\prime \prime}\right)\left(\mathfrak{x}_{2}^{\prime \prime} \diamond \mathfrak{x}^{\prime \prime \prime}\right) .
$$

In the same way, we have

$$
\left(\mathfrak{x}^{\prime} \diamond \mathfrak{x}_{1}^{\prime \prime}\right)\left(\mathfrak{x}_{2}^{\prime \prime} \diamond \mathfrak{x}^{\prime \prime \prime}\right)=\mathfrak{x}^{\prime} \diamond\left(\mathfrak{x}^{\prime \prime} \diamond \mathfrak{x}^{\prime \prime \prime}\right)
$$

This proves the associativity.

In summary, the proof of the associativity has been reduced to the special case when $\mathfrak{x}^{\prime}, \mathfrak{x}^{\prime \prime}, \mathfrak{x}^{\prime \prime \prime} \in \mathfrak{X}_{\infty}$ are chosen so that

(a) $n=\operatorname{dep}\left(\mathfrak{x}^{\prime}\right)+\operatorname{dep}\left(\mathfrak{x}^{\prime \prime}\right)+\operatorname{dep}\left(\mathfrak{x}^{\prime \prime \prime}\right)=k+1 \geq 1$ with the assumption that the associativity holds when $n \leq k$.

(b) the elements have breadth one and

(c) $t\left(\mathfrak{x}^{\prime}\right)=h\left(\mathfrak{x}^{\prime \prime}\right)$ and $t\left(\mathfrak{x}^{\prime \prime}\right)=h\left(\mathfrak{x}^{\prime \prime \prime}\right)$.

By Item (b), the head and tail of each of the elements are the same. Therefore by Item (c), either all the three elements are in $X$ or they are all in $\left\lfloor\mathfrak{X}_{\infty}\right\rfloor$. If all of $\mathfrak{x}^{\prime}, \mathfrak{x}^{\prime \prime}, \mathfrak{x}^{\prime \prime \prime}$ are in $X$, then as already shown, the associativity follows from the associativity in $A$. So it remains to consider the case when $\mathfrak{x}^{\prime}, \mathfrak{x}^{\prime \prime}, \mathfrak{x}^{\prime \prime \prime}$ are all in $\left\lfloor\mathfrak{X}_{\infty}\right\rfloor$. Then we may write

$$
\mathfrak{x}^{\prime}=\left\lfloor\overline{\mathfrak{x}}^{\prime}\right\rfloor, \mathfrak{x}^{\prime \prime}=\left\lfloor\overline{\mathfrak{x}}^{\prime \prime}\right\rfloor, \mathfrak{x}^{\prime \prime \prime}=\left\lfloor\overline{\mathfrak{x}}^{\prime \prime \prime}\right\rfloor \text { with } \overline{\mathfrak{x}}^{\prime}, \overline{\mathfrak{x}}^{\prime \prime}, \overline{\mathfrak{x}}^{\prime \prime \prime} \in \mathfrak{X}_{\infty} .
$$

Applying Eq. (7) and bilinearity of the product $\diamond$, we get

$$
\begin{aligned}
\left(\mathfrak{x}^{\prime} \diamond \mathfrak{x}^{\prime \prime}\right) \diamond \mathfrak{x}^{\prime \prime \prime}= & \left(\left\lfloor\left\lfloor\overline{\mathfrak{x}}^{\prime}\right\rfloor \diamond \overline{\mathfrak{x}}^{\prime \prime}\right\rfloor+\left\lfloor\overline{\mathfrak{x}}^{\prime} \diamond\left\lfloor\overline{\mathfrak{x}}^{\prime \prime}\right\rfloor\right\rfloor+\kappa \overline{\mathfrak{x}}^{\prime} \diamond \overline{\mathfrak{x}}^{\prime \prime}\right) \diamond\left\lfloor\overline{\mathfrak{x}}^{\prime \prime \prime}\right\rfloor \\
= & \left\lfloor\left\lfloor\overline{\mathfrak{x}}^{\prime}\right\rfloor \diamond \overline{\mathfrak{x}}^{\prime \prime}\right\rfloor \diamond\left\lfloor\overline{\mathfrak{x}}^{\prime \prime \prime}\right\rfloor+\left\lfloor\overline{\mathfrak{x}}^{\prime} \diamond\left\lfloor\overline{\mathfrak{x}}^{\prime \prime}\right\rfloor\right\rfloor \diamond\left\lfloor\overline{\mathfrak{x}}^{\prime \prime \prime}\right\rfloor+\kappa\left(\overline{\mathfrak{x}}^{\prime} \diamond \overline{\mathfrak{x}}^{\prime \prime}\right) \diamond\left\lfloor\overline{\mathfrak{x}}^{\prime \prime \prime}\right\rfloor \\
= & \left.\left\lfloor\left\lfloor\overline{\mathfrak{x}}^{\prime}\right\rfloor \diamond \overline{\mathfrak{x}}^{\prime \prime}\right\rfloor \diamond \overline{\mathfrak{x}}^{\prime \prime \prime}\right\rfloor+\left\lfloor\left(\left\lfloor\overline{\mathfrak{x}}^{\prime}\right\rfloor \diamond \overline{\mathfrak{x}}^{\prime \prime}\right) \diamond\left\lfloor\overline{\mathfrak{x}}^{\prime \prime \prime}\right\rfloor\right\rfloor+\kappa\left(\left\lfloor\overline{\mathfrak{x}}^{\prime}\right\rfloor \diamond \overline{\mathfrak{x}}^{\prime \prime}\right) \diamond \overline{\mathfrak{x}}^{\prime \prime \prime} \\
& +\left\lfloor\left\lfloor\overline{\mathfrak{x}}^{\prime} \diamond\left\lfloor\overline{\mathfrak{x}}^{\prime \prime}\right\rfloor\right\rfloor \diamond \overline{\mathfrak{x}}^{\prime \prime \prime}\right\rfloor+\left\lfloor\left(\overline{\mathfrak{x}}^{\prime} \diamond\left\lfloor\overline{\mathfrak{x}}^{\prime \prime}\right\rfloor\right) \diamond\left\lfloor\overline{\mathfrak{x}}^{\prime \prime \prime}\right\rfloor\right\rfloor+\kappa\left(\overline{\mathfrak{x}}^{\prime} \diamond\left\lfloor\overline{\mathfrak{x}}^{\prime \prime}\right\rfloor\right) \diamond \overline{\mathfrak{x}}^{\prime \prime \prime} \\
& +\kappa\left(\overline{\mathfrak{x}}^{\prime} \diamond \overline{\mathfrak{x}}^{\prime \prime}\right) \diamond\left\lfloor\overline{\mathfrak{x}}^{\prime \prime \prime}\right\rfloor \\
= & \left.\left\lfloor\left\lfloor\overline{\mathfrak{x}}^{\prime}\right\rfloor \diamond \overline{\mathfrak{x}}^{\prime \prime}\right\rfloor \diamond \overline{\mathfrak{x}}^{\prime \prime \prime}\right\rfloor+\left\lfloor\left(\left\lfloor\overline{\mathfrak{x}}^{\prime}\right\rfloor \diamond \overline{\mathfrak{x}}^{\prime \prime}\right) \diamond\left\lfloor\overline{\mathfrak{x}}^{\prime \prime \prime}\right\rfloor\right\rfloor+\kappa\left(\left\lfloor\overline{\mathfrak{x}}^{\prime}\right\rfloor \diamond \overline{\mathfrak{x}}^{\prime \prime}\right) \diamond \overline{\mathfrak{x}}^{\prime \prime \prime} \\
& \left.\left.+\left\lfloor\left\lfloor\overline{\mathfrak{x}}^{\prime} \diamond\left\lfloor\overline{\mathfrak{x}}^{\prime \prime}\right\rfloor\right\rfloor \diamond \overline{\mathfrak{x}}^{\prime \prime \prime}\right\rfloor\right\rfloor+\left\lfloor\overline{\mathfrak{x}}^{\prime} \diamond\left\lfloor\overline{\mathfrak{x}}^{\prime \prime}\right\rfloor \diamond \overline{\mathfrak{x}}^{\prime \prime \prime}\right\rfloor\right\rfloor+\left\lfloor\overline{\mathfrak{x}}^{\prime} \diamond\left\lfloor\overline{\mathfrak{x}}^{\prime \prime} \diamond\left\lfloor\overline{\mathfrak{x}}^{\prime \prime \prime}\right\rfloor\right\rfloor\right. \\
& +\kappa\left\lfloor\overline{\mathfrak{x}}^{\prime} \diamond \overline{\mathfrak{x}}^{\prime \prime} \diamond \overline{\mathfrak{x}}^{\prime \prime \prime}\right\rfloor+\kappa\left(\overline{\mathfrak{x}}^{\prime} \diamond\left\lfloor\overline{\mathfrak{x}}^{\prime \prime}\right\rfloor\right) \diamond \overline{\mathfrak{x}}^{\prime \prime \prime}+\kappa\left(\overline{\mathfrak{x}}^{\prime} \diamond \overline{\mathfrak{x}}^{\prime \prime}\right) \diamond\left\lfloor\overline{\mathfrak{x}}^{\prime \prime \prime}\right\rfloor .
\end{aligned}
$$

Similarly we obtain

$$
\begin{aligned}
\mathfrak{x}^{\prime} \diamond\left(\mathfrak{x}^{\prime \prime} \diamond \mathfrak{x}^{\prime \prime \prime}\right) & =\left\lfloor\overline{\mathfrak{x}}^{\prime}\right\rfloor \diamond\left(\left\lfloor\left\lfloor\overline{\mathfrak{x}}^{\prime \prime}\right\rfloor \diamond \overline{\mathfrak{x}}^{\prime \prime \prime}\right\rfloor+\left\lfloor\overline{\mathfrak{x}}^{\prime \prime} \diamond\left\lfloor\overline{\mathfrak{x}}^{\prime \prime \prime}\right\rfloor\right\rfloor+\kappa \overline{\mathfrak{x}}^{\prime \prime} \diamond \overline{\mathfrak{x}}^{\prime \prime \prime}\right) \\
& \left.=\left\lfloor\overline{\mathfrak{x}}^{\prime}\right\rfloor \diamond\left\lfloor\overline{\mathfrak{x}}^{\prime \prime}\right\rfloor \diamond \overline{\mathfrak{x}}^{\prime \prime \prime}\right\rfloor+\left\lfloor\overline{\mathfrak{x}}^{\prime}\right\rfloor \diamond\left\lfloor\overline{\mathfrak{x}}^{\prime \prime} \diamond\left\lfloor\overline{\mathfrak{x}}^{\prime \prime \prime}\right\rfloor\right\rfloor+\kappa\left\lfloor\overline{\mathfrak{x}}^{\prime}\right\rfloor \diamond\left(\overline{\mathfrak{x}}^{\prime \prime} \diamond \overline{\mathfrak{x}}^{\prime \prime \prime}\right)
\end{aligned}
$$




$$
\begin{aligned}
= & \left.\left\lfloor\left\lfloor\overline{\mathfrak{x}}^{\prime}\right\rfloor \diamond\left(\left\lfloor\overline{\mathfrak{x}}^{\prime \prime}\right\rfloor \diamond \overline{\mathfrak{x}}^{\prime \prime \prime}\right)\right\rfloor+\left\lfloor\overline{\mathfrak{x}}^{\prime} \diamond\left\lfloor\overline{\mathfrak{x}}^{\prime \prime}\right\rfloor \diamond \overline{\mathfrak{x}}^{\prime \prime \prime}\right\rfloor\right\rfloor+\kappa \overline{\mathfrak{x}}^{\prime} \diamond\left(\left\lfloor\overline{\mathfrak{x}}^{\prime \prime}\right\rfloor \diamond \overline{\mathfrak{x}}^{\prime \prime \prime}\right) \\
& +\left\lfloor\left\lfloor\overline{\mathfrak{x}}^{\prime}\right\rfloor \diamond\left(\overline{\mathfrak{x}}^{\prime \prime} \diamond\left\lfloor\overline{\mathfrak{x}}^{\prime \prime \prime}\right\rfloor\right)\right\rfloor+\left\lfloor\overline{\mathfrak{x}}^{\prime} \diamond\left\lfloor\overline{\mathfrak{x}}^{\prime \prime} \diamond\left\lfloor\overline{\mathfrak{x}}^{\prime \prime \prime}\right\rfloor\right\rfloor\right\rfloor+\kappa \overline{\mathfrak{x}}^{\prime} \diamond\left(\overline{\mathfrak{x}}^{\prime \prime} \diamond\left\lfloor\overline{\mathfrak{x}}^{\prime \prime \prime}\right\rfloor\right) \\
& +\kappa\left\lfloor\overline{\mathfrak{x}}^{\prime}\right\rfloor \diamond\left(\overline{\mathfrak{x}}^{\prime \prime} \diamond \overline{\mathfrak{x}}^{\prime \prime \prime}\right) \\
= & \left.\left\lfloor\left\lfloor\left\lfloor\overline{\mathfrak{x}}^{\prime}\right\rfloor \diamond \overline{\mathfrak{x}}^{\prime \prime}\right\rfloor \diamond \overline{\mathfrak{x}}^{\prime \prime \prime}\right\rfloor+\left\lfloor\overline{\mathfrak{x}}^{\prime} \diamond\left\lfloor\overline{\mathfrak{x}}^{\prime \prime}\right\rfloor\right\rfloor \diamond \overline{\mathfrak{x}}^{\prime \prime \prime}\right\rfloor \\
& \left.+\kappa\left\lfloor\overline{\mathfrak{x}}^{\prime} \diamond \overline{\mathfrak{x}}^{\prime \prime} \diamond \overline{\mathfrak{x}}^{\prime \prime \prime}\right\rfloor+\left\lfloor\overline{\mathfrak{x}}^{\prime} \diamond\left\lfloor\overline{\mathfrak{x}}^{\prime \prime}\right\rfloor \diamond \overline{\mathfrak{x}}^{\prime \prime \prime}\right\rfloor\right\rfloor+\kappa \overline{\mathfrak{x}}^{\prime} \diamond\left(\left\lfloor\overline{\mathfrak{x}}^{\prime \prime}\right\rfloor \diamond \overline{\mathfrak{x}}^{\prime \prime \prime}\right) \\
& +\left\lfloor\left\lfloor\overline{\mathfrak{x}}^{\prime}\right\rfloor \diamond\left(\overline{\mathfrak{x}}^{\prime \prime} \diamond\left\lfloor\overline{\mathfrak{x}}^{\prime \prime \prime}\right\rfloor\right)\right\rfloor+\left\lfloor\overline{\mathfrak{x}}^{\prime} \diamond\left\lfloor\overline{\mathfrak{x}}^{\prime \prime} \diamond\left\lfloor\overline{\mathfrak{x}}^{\prime \prime \prime \prime}\right\rfloor\right\rfloor\right\rfloor+\kappa \overline{\mathfrak{x}}^{\prime} \diamond\left(\overline{\mathfrak{x}}^{\prime \prime} \diamond\left\lfloor\overline{\mathfrak{x}}^{\prime \prime \prime}\right\rfloor\right) \\
& +\kappa\left\lfloor\overline{\mathfrak{x}}^{\prime}\right\rfloor \diamond\left(\overline{\mathfrak{x}}^{\prime \prime} \diamond \overline{\mathfrak{x}}^{\prime \prime \prime}\right) .
\end{aligned}
$$

Now by the induction hypothesis, the $i$-th term in the expansion of $\left(\mathfrak{x}^{\prime} \diamond \mathfrak{x}^{\prime \prime}\right) \diamond \mathfrak{x}^{\prime \prime \prime}$ coincides with the $\sigma(i)$-th term in the expansion of $\mathfrak{x}^{\prime} \diamond\left(\mathfrak{x}^{\prime \prime} \diamond \mathfrak{x}^{\prime \prime \prime}\right)$. Here $\sigma \in \Sigma_{9}$ is the permutation given by

$$
\sigma=\left(\begin{array}{ccccccccc}
1 & 2 & 3 & 4 & 5 & 6 & 7 & 8 & 9 \\
1 & 6 & 9 & 2 & 4 & 7 & 3 & 5 & 8
\end{array}\right) .
$$

This completes the proof of Theorem 2.6 (a).

(b). The proof follows from the definition $P_{A}(\mathfrak{x})=\lfloor\mathfrak{x}\rfloor$ and Eq. (7).

(c). Let $(M, *, P)$ be a modified Rota-Baxter algebra with multiplication $*$ and let $f: A \rightarrow M$ be a $\mathbf{k}$-algebra homomorphism. We will construct a $\mathbf{k}$-linear map $\bar{f}: F_{\kappa}(A) \rightarrow M$ by defining $\bar{f}(\mathfrak{x})$ for $\mathfrak{x} \in \mathfrak{X}_{\infty}$. We achieve this by defining $\bar{f}(\mathfrak{x})$ for $\mathfrak{x} \in \mathfrak{X}_{n}, n \geq 0$, inductively on $n$. For $\mathfrak{x} \in \mathfrak{X}_{0}:=X$, define $\bar{f}(\mathfrak{x})=f(\mathfrak{x})$. Then $j \bar{f}=f$ is satisfied. Suppose $\bar{f}(\mathfrak{x})$ has been defined for $\mathfrak{x} \in \mathfrak{X}_{n}$ and consider $\mathfrak{x}$ in $\mathfrak{X}_{n+1}$ which is, by definition,

$$
\begin{aligned}
\Lambda\left(X, \mathfrak{X}_{n}\right)= & \left(\bigsqcup_{r \geq 1}\left(X\left\lfloor\mathfrak{X}_{n}\right\rfloor\right)^{r}\right) \bigsqcup\left(\bigsqcup_{r \geq 0}\left(X\left\lfloor\mathfrak{X}_{n}\right\rfloor\right)^{r} X\right) \\
& \bigsqcup\left(\bigsqcup_{r \geq 0}\left\lfloor\mathfrak{X}_{n}\right\rfloor\left(X\left\lfloor\mathfrak{X}_{n}\right\rfloor\right)^{r}\right) \bigsqcup\left(\bigsqcup_{r \geq 0}\left\lfloor\mathfrak{X}_{n}\right\rfloor\left(X\left\lfloor\mathfrak{X}_{n}\right\rfloor\right)^{r} X\right) .
\end{aligned}
$$

Let $\mathfrak{x}$ be in the first union component $\bigsqcup_{r \geq 1}\left(X\left\lfloor\mathfrak{X}_{n}\right\rfloor\right)^{r}$ above. Then

$$
\mathfrak{x}=\prod_{i=1}^{r}\left(\mathfrak{x}_{2 i-1}\left\lfloor\mathfrak{x}_{2 i}\right\rfloor\right)
$$

for $\mathfrak{x}_{2 i-1} \in X$ and $\mathfrak{x}_{2 i} \in \mathfrak{X}_{n}, 1 \leq i \leq r$. By the construction of the multiplication $\diamond$ and the modified Rota-Baxter operator $P_{A}$, we have

$$
\mathfrak{x}=\diamond_{i=1}^{r}\left(\mathfrak{x}_{2 i-1} \diamond\left\lfloor\mathfrak{x}_{2 i}\right\rfloor\right)=\diamond_{i=1}^{r}\left(\mathfrak{x}_{2 i-1} \diamond P_{A}\left(\mathfrak{x}_{2 i}\right)\right) .
$$

Define

$$
\bar{f}(\mathfrak{x})=*_{i=1}^{r}\left(\bar{f}\left(\mathfrak{x}_{2 i-1}\right) * P_{A}\left(\bar{f}\left(\mathfrak{x}_{2 i}\right)\right)\right) .
$$


where the right hand side is well-defined by the induction hypothesis. Similarly define $\bar{f}(\mathfrak{x})$ if $\mathfrak{x}$ is in the other union components. For any $\mathfrak{x} \in \mathfrak{X}_{\infty}$, we have $P_{A}(\mathfrak{x})=$ $\lfloor\mathfrak{x}\rfloor \in \mathfrak{X}_{\infty}$, and by the definition of $\bar{f}$ in (Eq. (10)), we have

$$
\bar{f}(\lfloor\mathfrak{x}\rfloor)=P(\bar{f}(\mathfrak{x})) .
$$

So $\bar{f}$ commutes with the modified Rota-Baxter operators. Combining this equation with Eq. (10) we see that if $\mathfrak{x}=\mathfrak{x}_{1} \cdots \mathfrak{x}_{b}$ is the standard decomposition of $\mathfrak{x}$, then

$$
\bar{f}(\mathfrak{x})=\bar{f}\left(\mathfrak{x}_{1}\right) * \cdots * \bar{f}\left(\mathfrak{x}_{b}\right) .
$$

Note that this is the only possible way to define $\bar{f}(\mathfrak{x})$ in order for $\bar{f}$ to be a modified Rota-Baxter algebra homomorphism extending $f$. It remains to prove that the map $\bar{f}$ defined in Eq. (10) is indeed an algebra homomorphism. For this we only need to check the multiplicity

$$
\bar{f}\left(\mathfrak{x} \diamond \mathfrak{x}^{\prime}\right)=\bar{f}(\mathfrak{x}) * \bar{f}\left(\mathfrak{x}^{\prime}\right)
$$

for all $\mathfrak{x}, \mathfrak{x}^{\prime} \in \mathfrak{X}_{\infty}$. For this we use induction on the sum of depths $n:=\operatorname{bre}(\mathfrak{x})+$ bre $\left(\mathfrak{x}^{\prime}\right)$. Then $n \geq 0$. When $n=0$, we have $\mathfrak{x}, \mathfrak{x}^{\prime} \in X$. Then Eq. (12) follows from the multiplicity of $f$. Assume the multiplicity holds for $\mathfrak{x}, \mathfrak{x}^{\prime} \in \mathfrak{X}_{\infty}$ with $n \geq k$ and take $\mathfrak{x}, \mathfrak{x}^{\prime} \in \mathfrak{X}_{\infty}$ with $n=k+1$. Let $\mathfrak{x}=\mathfrak{x}_{1} \cdots \mathfrak{x}_{b}$ and $\mathfrak{x}^{\prime}=\mathfrak{x}_{1}^{\prime} \cdots \mathfrak{x}_{b^{\prime}}^{\prime}$ be the standard decompositions. Since $n=k+1 \geq 1$, at least one of $\mathfrak{x}_{b}$ and $\mathfrak{x}_{b^{\prime}}^{\prime}$ is in $\left\lfloor\mathfrak{X}_{\infty}\right\rfloor$. Then by Eq. (7) we have

$\bar{f}\left(\mathfrak{x}_{b} \diamond \mathfrak{x}_{1}^{\prime}\right)= \begin{cases}\bar{f}\left(\mathfrak{x}_{b} \mathfrak{x}_{1}^{\prime}\right), & \text { if } \mathfrak{x}_{b} \in X, \mathfrak{x}_{1}^{\prime} \in\left\lfloor\mathfrak{X}_{\infty}\right\rfloor, \\ \bar{f}\left(\mathfrak{x}_{b} \mathfrak{x}_{1}^{\prime}\right), & \text { if } \mathfrak{x}_{b} \in\left\lfloor\mathfrak{X}_{\infty}\right\rfloor, \mathfrak{x}_{1}^{\prime} \in X, \\ \bar{f}\left(\left\lfloor\left\lfloor\overline{\mathfrak{x}}_{b}\right\rfloor \diamond \overline{\mathfrak{x}}_{1}^{\prime}\right\rfloor+\left\lfloor\overline{\mathfrak{x}}_{b} \diamond\left\lfloor\overline{\mathfrak{x}}_{1}^{\prime}\right\rfloor\right\rfloor+\kappa \overline{\mathfrak{x}}_{b} \diamond \overline{\mathfrak{x}}_{1}^{\prime}\right), & \text { if } \mathfrak{x}_{b}=\left\lfloor\overline{\mathfrak{x}}_{b}\right\rfloor, \mathfrak{x}_{1}^{\prime}=\left\lfloor\overline{\mathfrak{x}}_{1}^{\prime}\right\rfloor \in\left\lfloor\mathfrak{X}_{\infty}\right\rfloor .\end{cases}$

In the first two cases, the right hand side is $\bar{f}\left(\mathfrak{x}_{b}\right) * \bar{f}\left(\mathfrak{x}_{1}^{\prime}\right)$ by the definition of $\bar{f}$. In the third case, applying Eq. (11), the induction hypothesis and the modified Rota-Baxter relation of the operator $P$ on $M$, we have

$$
\begin{aligned}
& \bar{f}\left(\left\lfloor\left\lfloor\overline{\mathfrak{x}}_{b}\right\rfloor \diamond \overline{\mathfrak{x}}_{1}^{\prime}\right\rfloor+\left\lfloor\overline{\mathfrak{x}}_{b} \diamond\left\lfloor\overline{\mathfrak{x}}_{1}^{\prime}\right\rfloor\right\rfloor+\kappa \overline{\mathfrak{x}}_{b} \diamond \overline{\mathfrak{x}}_{1}^{\prime}\right) \\
= & \bar{f}\left(\left\lfloor\left\lfloor\overline{\mathfrak{x}}_{b}\right\rfloor \diamond \overline{\mathfrak{x}}_{1}^{\prime}\right\rfloor\right)+\bar{f}\left(\left\lfloor\overline{\mathfrak{x}}_{b} \diamond\left\lfloor\overline{\mathfrak{x}}_{1}^{\prime}\right\rfloor\right\rfloor\right)+\kappa \bar{f}\left(\overline{\mathfrak{x}}_{b} \diamond \overline{\mathfrak{x}}_{1}^{\prime}\right) \\
= & P\left(\bar{f}\left(\left\lfloor\overline{\mathfrak{x}}_{b}\right\rfloor \diamond \overline{\mathfrak{x}}_{1}^{\prime}\right)\right)+P\left(\bar{f}\left(\overline{\mathfrak{x}}_{b} \diamond\left\lfloor\overline{\mathfrak{x}}_{1}^{\prime}\right\rfloor\right)\right)+\kappa \bar{f}\left(\overline{\mathfrak{x}}_{b} \diamond \overline{\mathfrak{x}}_{1}^{\prime}\right) \\
= & P\left(\bar{f}\left(\left\lfloor\overline{\mathfrak{x}}_{b}\right\rfloor\right) * \bar{f}\left(\overline{\mathfrak{x}}_{1}^{\prime}\right)\right)+P\left(\bar{f}\left(\overline{\mathfrak{x}}_{b}\right) * \bar{f}\left(\left\lfloor\overline{\mathfrak{x}}_{1}^{\prime}\right\rfloor\right)\right)+\kappa \bar{f}\left(\overline{\mathfrak{x}}_{b}\right) * \bar{f}\left(\overline{\mathfrak{x}}_{1}^{\prime}\right) \\
= & P\left(P\left(\bar{f}\left(\overline{\mathfrak{x}}_{b}\right)\right) * \bar{f}\left(\overline{\mathfrak{x}}_{1}^{\prime}\right)\right)+P\left(\bar{f}\left(\overline{\mathfrak{x}}_{b}\right) * P\left(\bar{f}\left(\overline{\mathfrak{x}}_{1}^{\prime}\right)\right)\right)+\kappa\left(\bar{f}\left(\overline{\mathfrak{x}}_{b}\right) * \bar{f}\left(\overline{\mathfrak{x}}_{1}^{\prime}\right)\right) \\
= & P\left(\bar{f}\left(\overline{\mathfrak{x}}_{b}\right)\right) * P\left(\bar{f}\left(\overline{\mathfrak{x}}_{1}^{\prime}\right)\right)
\end{aligned}
$$




$$
\begin{aligned}
& =\bar{f}\left(\left\lfloor\overline{\mathfrak{x}}_{b}\right\rfloor\right) * \bar{f}\left(\left\lfloor\overline{\mathfrak{x}}_{1}^{\prime}\right\rfloor\right) \\
& =\bar{f}\left(\mathfrak{x}_{b}\right) * \bar{f}\left(\mathfrak{x}_{1}^{\prime}\right) .
\end{aligned}
$$

Therefore $\bar{f}\left(\mathfrak{x}_{b} \diamond \mathfrak{x}_{1}^{\prime}\right)=\bar{f}\left(\mathfrak{x}_{b}\right) * \bar{f}\left(\mathfrak{x}_{1}^{\prime}\right)$. Then

$$
\begin{aligned}
\bar{f}\left(\mathfrak{x} \diamond \mathfrak{x}^{\prime}\right) & =\bar{f}\left(\mathfrak{x}_{1} \cdots \mathfrak{x}_{b-1}\left(\mathfrak{x}_{b} \diamond \mathfrak{x}_{1}^{\prime}\right) \mathfrak{x}_{2}^{\prime} \cdots \mathfrak{x}_{b^{\prime}}^{\prime}\right) \\
& =\bar{f}\left(\mathfrak{x}_{1}\right) * \cdots * \bar{f}\left(\mathfrak{x}_{b-1}\right) * \bar{f}\left(\mathfrak{x}_{b} \diamond \mathfrak{x}_{1}^{\prime}\right) * \bar{f}\left(\mathfrak{x}_{2}^{\prime}\right) \cdots \bar{f}\left(\mathfrak{x}_{b^{\prime}}^{\prime}\right) \\
& =\bar{f}\left(\mathfrak{x}_{1}\right) * \cdots * \bar{f}\left(\mathfrak{x}_{b-1}\right) * \bar{f}\left(\mathfrak{x}_{b}\right) * \bar{f}\left(\mathfrak{x}_{1}^{\prime}\right) * \bar{f}\left(\mathfrak{x}_{2}^{\prime}\right) \cdots \bar{f}\left(\mathfrak{x}_{b^{\prime}}^{\prime}\right) \\
& =\bar{f}(\mathfrak{x}) * \bar{f}\left(\mathfrak{x}^{\prime}\right),
\end{aligned}
$$

as required.

This completes the proof of Theorem 2.6

\section{The Hopf algebra structure on free modified Rota-Baxter algebras}

In this section, starting with the assumption that $A$ is a bialgebra with its coproduct $\Delta_{A}$ and its counit $\varepsilon_{A}$, we provide a bialgebraic and then a Hopf algebraic structure on the free modified Rota-Baxter algebras $F_{\kappa}(A)$ obtained in Section 2, when $\kappa=-\lambda^{2}$. It would be interesting to see how to extend this construction to other weights $\kappa$. For Hopf algebra structures on free Rota-Baxter algebras, see $[15,28]$ for Hopf algebra structures on free Rota-Baxter algebras.

3.1. The bialgebraic structure. We now build on results from previous subsections to obtain a bialgebra structure on $F_{-\lambda^{2}}(A)$. We first record some lemmas for a preparation.

Lemma 3.1. Let $\lambda$ be a given element of $\mathbf{k}$.

(a) The linear map $-\lambda \mathrm{id}: \mathbf{k} \rightarrow \mathbf{k}$ is a modified Rota-Baxter operator of weight $-\lambda^{2}$ on $\mathbf{k}$.

(b) There exists a unique modified Rota-Baxter algebra morphism $\varepsilon_{\mathrm{M}}: F_{-\lambda^{2}}(A) \rightarrow$ $\mathbf{k}$ such that

$$
\varepsilon_{\mathrm{M}} \circ j_{A}=\varepsilon_{A} \text { and } \varepsilon_{\mathrm{M}} \circ P_{A}=-\lambda \mathrm{id} \circ \varepsilon_{\mathrm{M}} .
$$

Proof. (a) It follows from

$$
\begin{aligned}
(-\lambda \mathrm{id})(a)(-\lambda \mathrm{id})(b) & =\lambda^{2} a b=\lambda^{2} a b+\lambda^{2} a b-\lambda^{2} a b \\
& =(-\lambda \mathrm{id})(a(-\lambda \mathrm{id})(b))+(-\lambda \mathrm{id})((-\lambda \mathrm{id})(a) b)-\lambda^{2} a b
\end{aligned}
$$


(b) By Item (a), (k, $-\lambda$ id) is a modified Rota-Baxter algebra of weight $-\lambda^{2}$. Then the remainder follows from Theorem 2.6 (c).

Note that $P_{A}$ is a modified Rota-Baxter operator on $F_{-\lambda^{2}}(A)$; however $P_{A} \otimes P_{A}$ is not a modified Rota-Baxter operator on $F_{-\lambda^{2}}(A) \otimes F_{-\lambda^{2}}(A)$. The following result constructs a modified Rota-Baxter operator on $F_{-\lambda^{2}}(A) \otimes F_{-\lambda^{2}}(A)$.

Lemma 3.2. Let $\lambda$ be a given element of $\mathbf{k}$. Define the linear map

$$
Q: F_{-\lambda^{2}}(A) \otimes F_{-\lambda^{2}}(A) \rightarrow F_{-\lambda^{2}}(A) \otimes F_{-\lambda^{2}}(A)
$$

by taking

$$
Q\left(\mathfrak{x} \otimes \mathfrak{x}^{\prime}\right):=\left(P_{A}(\mathfrak{x})+\lambda \mathfrak{x}\right) \otimes \varepsilon_{\mathrm{M}}\left(\mathfrak{x}^{\prime}\right) 1+\mathfrak{x} \otimes P_{A}\left(\mathfrak{x}^{\prime}\right) \text { for } \mathfrak{x}, \mathfrak{x}^{\prime} \in F_{-\lambda^{2}}(A) .
$$

Then $Q$ is a modified Rota-Baxter operator of weight $-\lambda^{2}$ on $F_{-\lambda^{2}}(A) \otimes F_{-\lambda^{2}}(A)$.

Proof. Let $\mathfrak{x}_{1}, \mathfrak{x}_{2}, \mathfrak{x}_{1}^{\prime}, \mathfrak{x}_{2}^{\prime} \in F_{-\lambda^{2}}(A)$. On the one hand,

$$
\begin{aligned}
& Q\left(\mathfrak{x}_{1} \otimes \mathfrak{x}_{1}^{\prime}\right) \diamond^{\prime} Q\left(\mathfrak{x}_{2} \otimes \mathfrak{x}_{2}^{\prime}\right) \\
= & \left(\left(P_{A}\left(\mathfrak{x}_{1}\right)+\lambda \mathfrak{x}_{1}\right) \otimes \varepsilon_{\mathrm{M}}\left(\mathfrak{x}_{1}^{\prime}\right) 1+\mathfrak{x}_{1} \otimes P_{A}\left(\mathfrak{x}_{1}^{\prime}\right)\right) \diamond^{\prime}\left(\left(P_{A}\left(\mathfrak{x}_{2}\right)+\lambda \mathfrak{x}_{2}\right) \otimes \varepsilon_{\mathrm{M}}\left(\mathfrak{x}_{2}^{\prime}\right) 1+\mathfrak{x}_{2} \otimes P_{A}\left(\mathfrak{x}_{2}^{\prime}\right)\right) \\
= & \left(\left(P_{A}\left(\mathfrak{x}_{1}\right)+\lambda \mathfrak{x}_{1}\right) \diamond\left(P_{A}\left(\mathfrak{x}_{2}\right)+\lambda \mathfrak{x}_{2}\right)\right) \otimes \varepsilon_{\mathrm{M}}\left(\mathfrak{x}_{1}^{\prime}\right) \varepsilon_{\mathrm{M}}\left(\mathfrak{x}_{2}^{\prime}\right) 1+\left(\left(P_{A}\left(\mathfrak{x}_{1}\right)+\lambda \mathfrak{x}_{1}\right) \diamond \mathfrak{x}_{2}\right) \otimes \varepsilon_{\mathrm{M}}\left(\mathfrak{x}_{1}^{\prime}\right) P_{A}\left(\mathfrak{x}_{2}^{\prime}\right) \\
& +\left(\mathfrak{x}_{1} \diamond\left(P_{A}\left(\mathfrak{x}_{2}\right)+\lambda \mathfrak{x}_{2}\right)\right) \otimes P_{A}\left(\mathfrak{x}_{1}^{\prime}\right) \varepsilon_{\mathrm{M}}\left(\mathfrak{x}_{2}^{\prime}\right)+\left(\mathfrak{x}_{1} \diamond \mathfrak{x}_{2}\right) \otimes\left(P_{A}\left(\mathfrak{x}_{1}^{\prime}\right) \diamond P_{A}\left(\mathfrak{x}_{2}^{\prime}\right)\right) \\
= & \left(P_{A}\left(\mathfrak{x}_{1}\right) \diamond P_{A}\left(\mathfrak{x}_{2}\right)+\lambda P_{A}\left(\mathfrak{x}_{1}\right) \diamond \mathfrak{x}_{2}+\lambda \mathfrak{x}_{1} \diamond P_{A}\left(\mathfrak{x}_{2}\right)+\lambda^{2} \mathfrak{x}_{1} \diamond \mathfrak{x}_{2}\right) \otimes \varepsilon_{\mathrm{M}}\left(\mathfrak{x}_{1}^{\prime}\right) \varepsilon_{\mathrm{M}}\left(\mathfrak{x}_{2}^{\prime}\right) 1 \\
& +\left(P_{A}\left(\mathfrak{x}_{1}\right) \diamond \mathfrak{x}_{2}\right) \otimes \varepsilon_{\mathrm{M}}\left(\mathfrak{x}_{1}^{\prime}\right) P_{A}\left(\mathfrak{x}_{2}^{\prime}\right)+\lambda\left(\mathfrak{x}_{1} \diamond \mathfrak{x}_{2}\right) \otimes \varepsilon_{\mathrm{M}}\left(\mathfrak{x}_{1}^{\prime}\right) P_{A}\left(\mathfrak{x}_{2}^{\prime}\right)+\left(\mathfrak{x}_{1} \diamond P_{A}\left(\mathfrak{x}_{2}\right)\right) \otimes P_{A}\left(\mathfrak{x}_{1}^{\prime}\right) \varepsilon_{\mathrm{M}}\left(\mathfrak{x}_{2}^{\prime}\right) \\
& +\lambda\left(\mathfrak{x}_{1} \diamond \mathfrak{x}_{2}\right) \otimes P_{A}\left(\mathfrak{x}_{1}^{\prime}\right) \varepsilon_{\mathrm{M}}\left(\mathfrak{x}_{2}^{\prime}\right)+\left(\mathfrak{x}_{1} \diamond \mathfrak{x}_{2}\right) \otimes\left(P_{A}\left(\mathfrak{x}_{1}^{\prime}\right) \diamond P_{A}\left(\mathfrak{x}_{2}^{\prime}\right)\right) \\
= & \left(P_{A}\left(\mathfrak{x}_{1} \diamond P_{A}\left(\mathfrak{x}_{2}\right)\right)+P_{A}\left(P_{A}\left(\mathfrak{x}_{1}\right) \diamond \mathfrak{x}_{2}\right)+\lambda P_{A}\left(\mathfrak{x}_{1}\right) \diamond \mathfrak{x}_{2}+\lambda \mathfrak{x}_{1} \diamond P_{A}\left(\mathfrak{x}_{2}\right)\right) \otimes \varepsilon_{\mathrm{M}}\left(\mathfrak{x}_{1}^{\prime}\right) \varepsilon_{\mathrm{M}}\left(\mathfrak{x}_{2}^{\prime}\right) 1 \\
& +\left(P_{A}\left(\mathfrak{x}_{1}\right) \diamond \mathfrak{x}_{2}\right) \otimes \varepsilon_{\mathrm{M}}\left(\mathfrak{x}_{1}^{\prime}\right) P_{A}\left(\mathfrak{x}_{2}^{\prime}\right)+\lambda\left(\mathfrak{x}_{1} \diamond \mathfrak{x}_{2}\right) \otimes \varepsilon_{\mathrm{M}}\left(\mathfrak{x}_{1}^{\prime}\right) P_{A}\left(\mathfrak{x}_{2}^{\prime}\right)+\left(\mathfrak{x}_{1} \diamond P_{A}\left(\mathfrak{x}_{2}\right)\right) \otimes P_{A}\left(\mathfrak{x}_{1}^{\prime}\right) \varepsilon_{\mathrm{M}}\left(\mathfrak{x}_{2}^{\prime}\right) \\
& +\lambda\left(\mathfrak{x}_{1} \diamond \mathfrak{x}_{2}\right) \otimes P_{A}\left(\mathfrak{x}_{1}^{\prime}\right) \varepsilon_{\mathrm{M}}\left(\mathfrak{x}_{2}^{\prime}\right)+\left(\mathfrak{x}_{1} \diamond \mathfrak{x}_{2}\right) \otimes\left(P_{A}\left(\mathfrak{x}_{1} \diamond P_{A}\left(\mathfrak{x}_{2}\right)\right)+P_{A}\left(P_{A}\left(\mathfrak{x}_{1}\right) \diamond \mathfrak{x}_{2}\right)-\lambda^{2} \mathfrak{x}_{1}^{\prime} \diamond \mathfrak{x}_{2}^{\prime}\right) .
\end{aligned}
$$

(by Theorem (2.6) (b))

On the other hand,

$$
\begin{aligned}
& Q\left(\left(\mathfrak{x}_{1} \otimes \mathfrak{x}_{1}^{\prime}\right) \diamond^{\prime} Q\left(\mathfrak{x}_{2} \otimes \mathfrak{x}_{2}^{\prime}\right)\right)+Q\left(Q\left(\mathfrak{x}_{1} \otimes \mathfrak{x}_{1}^{\prime}\right) \diamond^{\prime}\left(\mathfrak{x}_{2} \otimes \mathfrak{x}_{2}^{\prime}\right)\right)-\lambda^{2}\left(\left(\mathfrak{x}_{1} \otimes \mathfrak{x}_{1}^{\prime}\right) \diamond^{\prime}\left(\mathfrak{x}_{2} \otimes \mathfrak{x}_{2}^{\prime}\right)\right) \\
= & Q\left(\left(\mathfrak{x}_{1} \otimes \mathfrak{x}_{1}^{\prime}\right) \diamond^{\prime}\left(\left(P_{A}\left(\mathfrak{x}_{2}\right)+\lambda \mathfrak{x}_{2}\right) \otimes \varepsilon_{\mathrm{M}}\left(\mathfrak{x}_{2}^{\prime}\right) 1+\mathfrak{x}_{2} \otimes P_{A}\left(\mathfrak{x}_{2}^{\prime}\right)\right)\right) \\
& +Q\left(\left(\left(P_{A}\left(\mathfrak{x}_{1}\right)+\lambda \mathfrak{x}_{1}\right) \otimes \varepsilon_{\mathrm{M}}\left(\mathfrak{x}_{1}^{\prime}\right) 1+\mathfrak{x}_{1} \otimes P_{A}\left(\mathfrak{x}_{1}^{\prime}\right)\right) \diamond^{\prime}\left(\mathfrak{x}_{2} \otimes \mathfrak{x}_{2}^{\prime}\right)\right)-\lambda^{2}\left(\mathfrak{x}_{1} \diamond \mathfrak{x}_{2}\right) \otimes\left(\mathfrak{x}_{1}^{\prime} \diamond \mathfrak{x}_{2}^{\prime}\right) \\
= & Q\left(\left(\mathfrak{x}_{1} \diamond P_{A}\left(\mathfrak{x}_{2}\right)\right) \otimes \mathfrak{x}_{1}^{\prime} \varepsilon_{\mathrm{M}}\left(\mathfrak{x}_{2}^{\prime}\right)+\lambda\left(\mathfrak{x}_{1} \diamond \mathfrak{x}_{2}\right) \otimes \mathfrak{x}_{1}^{\prime} \varepsilon_{\mathrm{M}}\left(\mathfrak{x}_{2}^{\prime}\right)+\left(\mathfrak{x}_{1} \diamond \mathfrak{x}_{2}\right) \otimes\left(\mathfrak{x}_{1}^{\prime} \diamond P_{A}\left(\mathfrak{x}_{2}^{\prime}\right)\right)\right)
\end{aligned}
$$




$$
\begin{aligned}
& +Q\left(\left(P_{A}\left(\mathfrak{x}_{1}\right) \diamond \mathfrak{x}_{2}\right) \otimes \varepsilon_{\mathrm{M}}\left(\mathfrak{x}_{1}^{\prime}\right) \mathfrak{x}_{2}^{\prime}+\lambda\left(\mathfrak{x}_{1} \diamond \mathfrak{x}_{2}\right) \otimes \varepsilon_{\mathrm{M}}\left(\mathfrak{x}_{1}^{\prime}\right) \mathfrak{x}_{2}^{\prime}+\left(\mathfrak{x}_{1} \diamond \mathfrak{x}_{2}\right) \otimes\left(P_{A}\left(\mathfrak{x}_{1}^{\prime}\right) \diamond \mathfrak{x}_{2}^{\prime}\right)\right) \\
& -\lambda^{2}\left(\mathfrak{x}_{1} \diamond \mathfrak{x}_{2}\right) \otimes\left(\mathfrak{x}_{1}^{\prime} \diamond \mathfrak{x}_{2}^{\prime}\right) \\
& =\left(P_{A}\left(\mathfrak{x}_{1} \diamond P_{A}\left(\mathfrak{x}_{2}\right)\right)+\lambda \mathfrak{x}_{1} \diamond P_{A}\left(\mathfrak{x}_{2}\right)\right) \otimes \varepsilon_{\mathrm{M}}\left(\mathfrak{x}_{1}^{\prime} \varepsilon_{\mathrm{M}}\left(\mathfrak{x}_{2}^{\prime}\right)\right) 1+\left(\mathfrak{x}_{1} \diamond P_{A}\left(\mathfrak{x}_{2}\right)\right) \otimes P_{A}\left(\mathfrak{x}_{1}^{\prime} \varepsilon_{\mathrm{M}}\left(\mathfrak{x}_{2}^{\prime}\right)\right) \\
& +\lambda\left(P_{A}\left(\mathfrak{x}_{1} \diamond \mathfrak{x}_{2}\right)+\lambda \mathfrak{x}_{1} \diamond \mathfrak{x}_{2}\right) \otimes \varepsilon_{\mathrm{M}}\left(\mathfrak{x}_{1}^{\prime} \varepsilon_{\mathrm{M}}\left(\mathfrak{x}_{2}^{\prime}\right)\right) 1+\lambda\left(\mathfrak{x}_{1} \diamond \mathfrak{x}_{2}\right) \otimes P_{A}\left(\mathfrak{x}_{1}^{\prime} \varepsilon_{\mathrm{M}}\left(\mathfrak{x}_{2}^{\prime}\right)\right) \\
& +\left(P_{A}\left(\mathfrak{x}_{1} \diamond \mathfrak{x}_{2}\right)+\lambda \mathfrak{x}_{1} \diamond \mathfrak{x}_{2}\right) \otimes \varepsilon_{\mathrm{M}}\left(\mathfrak{x}_{1}^{\prime} P_{A}\left(\mathfrak{x}_{2}^{\prime}\right)\right) 1+\left(\mathfrak{x}_{1} \diamond \mathfrak{x}_{2}\right) \otimes P_{A}\left(\mathfrak{x}_{1}^{\prime} P_{A}\left(\mathfrak{x}_{2}^{\prime}\right)\right) \\
& +\left(P_{A}\left(P_{A}\left(\mathfrak{x}_{1}\right) \diamond \mathfrak{x}_{2}\right)+\lambda P_{A}\left(\mathfrak{x}_{1}\right) \diamond \mathfrak{x}_{2}\right) \otimes \varepsilon_{\mathrm{M}}\left(\varepsilon_{\mathrm{M}}\left(\mathfrak{x}_{1}^{\prime}\right) \mathfrak{x}_{2}^{\prime}\right) 1+\left(P_{A}\left(\mathfrak{x}_{1}\right) \diamond \mathfrak{x}_{2}\right) \otimes P_{A}\left(\varepsilon_{\mathrm{M}}\left(\mathfrak{x}_{1}^{\prime}\right) \mathfrak{x}_{2}^{\prime}\right) \\
& +\lambda\left(P_{A}\left(\mathfrak{x}_{1} \diamond \mathfrak{x}_{2}\right)+\lambda \mathfrak{x}_{1} \diamond \mathfrak{x}_{2}\right) \otimes \varepsilon_{\mathrm{M}}\left(\varepsilon_{\mathrm{M}}\left(\mathfrak{x}_{1}^{\prime}\right) \mathfrak{x}_{2}^{\prime}\right) 1+\lambda\left(\mathfrak{x}_{1} \diamond \mathfrak{x}_{2}\right) \otimes P_{A}\left(\varepsilon_{\mathrm{M}}\left(\mathfrak{x}_{1}^{\prime}\right) \mathfrak{x}_{2}^{\prime}\right) \\
& +\left(P_{A}\left(\mathfrak{x}_{1} \diamond \mathfrak{x}_{2}\right)+\lambda \mathfrak{x}_{1} \diamond \mathfrak{x}_{2}\right) \otimes \varepsilon_{\mathrm{M}}\left(P_{A}\left(\mathfrak{x}_{1}^{\prime}\right) \mathfrak{x}_{2}^{\prime}\right)+\left(\mathfrak{x}_{1} \diamond \mathfrak{x}_{2}\right) \otimes P_{A}\left(P_{A}\left(\mathfrak{x}_{1}^{\prime}\right) \mathfrak{x}_{2}^{\prime}\right)-\lambda^{2}\left(\mathfrak{x}_{1} \diamond \mathfrak{x}_{2}\right) \otimes\left(\mathfrak{x}_{1}^{\prime} \diamond \mathfrak{x}_{2}^{\prime}\right) \\
& =\left(P_{A}\left(\mathfrak{x}_{1} \diamond P_{A}\left(\mathfrak{x}_{2}\right)\right)+\lambda \mathfrak{x}_{1} \diamond P_{A}\left(\mathfrak{x}_{2}\right)\right) \otimes \varepsilon_{\mathrm{M}}\left(\mathfrak{x}_{1}^{\prime}\right) \varepsilon_{\mathrm{M}}\left(\mathfrak{x}_{2}^{\prime}\right) 1+\left(\mathfrak{x}_{1} \diamond P_{A}\left(\mathfrak{x}_{2}\right)\right) \otimes P_{A}\left(\mathfrak{x}_{1}^{\prime}\right) \varepsilon_{\mathrm{M}}\left(\mathfrak{x}_{2}^{\prime}\right) \\
& +\lambda\left(P_{A}\left(\mathfrak{x}_{1} \diamond \mathfrak{x}_{2}\right)+\lambda \mathfrak{x}_{1} \diamond \mathfrak{x}_{2}\right) \otimes \varepsilon_{\mathrm{M}}\left(\mathfrak{x}_{1}^{\prime}\right) \varepsilon_{\mathrm{M}}\left(\mathfrak{x}_{2}^{\prime}\right) 1+\lambda\left(\mathfrak{x}_{1} \diamond \mathfrak{x}_{2}\right) \otimes P_{A}\left(\mathfrak{x}_{1}^{\prime}\right) \varepsilon_{\mathrm{M}}\left(\mathfrak{x}_{2}^{\prime}\right) \\
& +\left(P_{A}\left(\mathfrak{x}_{1} \diamond \mathfrak{x}_{2}\right)+\lambda \mathfrak{x}_{1} \diamond \mathfrak{x}_{2}\right) \otimes \varepsilon_{\mathrm{M}}\left(\mathfrak{x}_{1}^{\prime}\right) \varepsilon_{\mathrm{M}}\left(P_{A}\left(\mathfrak{x}_{2}^{\prime}\right)\right) 1+\left(\mathfrak{x}_{1} \diamond \mathfrak{x}_{2}\right) \otimes P_{A}\left(\mathfrak{x}_{1}^{\prime} P_{A}\left(\mathfrak{x}_{2}^{\prime}\right)\right) \\
& +\left(P_{A}\left(P_{A}\left(\mathfrak{x}_{1}\right) \diamond \mathfrak{x}_{2}\right)+\lambda P_{A}\left(\mathfrak{x}_{1}\right) \diamond \mathfrak{x}_{2}\right) \otimes \varepsilon_{\mathrm{M}}\left(\mathfrak{x}_{1}^{\prime}\right) \varepsilon_{\mathrm{M}}\left(\mathfrak{x}_{2}^{\prime}\right) 1+\left(P_{A}\left(\mathfrak{x}_{1}\right) \diamond \mathfrak{x}_{2}\right) \otimes \varepsilon_{\mathrm{M}}\left(\mathfrak{x}_{1}^{\prime}\right) P_{A}\left(\mathfrak{x}_{2}^{\prime}\right) \\
& +\lambda\left(P_{A}\left(\mathfrak{x}_{1} \diamond \mathfrak{x}_{2}\right)+\lambda \mathfrak{x}_{1} \diamond \mathfrak{x}_{2}\right) \otimes \varepsilon_{\mathrm{M}}\left(\mathfrak{x}_{1}^{\prime}\right) \varepsilon_{\mathrm{M}}\left(\mathfrak{x}_{2}^{\prime}\right) 1+\lambda\left(\mathfrak{x}_{1} \diamond \mathfrak{x}_{2}\right) \otimes \varepsilon_{\mathrm{M}}\left(\mathfrak{x}_{1}^{\prime}\right) P_{A}\left(\mathfrak{x}_{2}^{\prime}\right) \\
& +\left(P_{A}\left(\mathfrak{x}_{1} \diamond \mathfrak{x}_{2}\right)+\lambda \mathfrak{x}_{1} \diamond \mathfrak{x}_{2}\right) \otimes \varepsilon_{\mathrm{M}}\left(P_{A}\left(\mathfrak{x}_{1}^{\prime}\right)\right) \varepsilon_{\mathrm{M}}\left(\mathfrak{x}_{2}^{\prime}\right) 1+\left(\mathfrak{x}_{1} \diamond \mathfrak{x}_{2}\right) \otimes P_{A}\left(P_{A}\left(\mathfrak{x}_{1}^{\prime}\right) \mathfrak{x}_{2}^{\prime}\right) \\
& -\lambda^{2}\left(\mathfrak{x}_{1} \diamond \mathfrak{x}_{2}\right) \otimes\left(\mathfrak{x}_{1}^{\prime} \diamond \mathfrak{x}_{2}^{\prime}\right) \quad \text { (by } \varepsilon_{\mathrm{M}} \text { is } \mathbf{k} \text {-linear and } \varepsilon_{\mathrm{M}} \text { is a homomorphism) } \\
& =\left(P_{A}\left(\mathfrak{x}_{1} \diamond P_{A}\left(\mathfrak{x}_{2}\right)\right)+\lambda \mathfrak{x}_{1} \diamond P_{A}\left(\mathfrak{x}_{2}\right)\right) \otimes \varepsilon_{\mathrm{M}}\left(\mathfrak{x}_{1}^{\prime}\right) \varepsilon_{\mathrm{M}}\left(\mathfrak{x}_{2}^{\prime}\right) 1+\left(\mathfrak{x}_{1} \diamond P_{A}\left(\mathfrak{x}_{2}\right)\right) \otimes P_{A}\left(\mathfrak{x}_{1}^{\prime}\right) \varepsilon_{\mathrm{M}}\left(\mathfrak{x}_{2}^{\prime}\right) \\
& +\lambda\left(P_{A}\left(\mathfrak{x}_{1} \diamond \mathfrak{x}_{2}\right)+\lambda \mathfrak{x}_{1} \diamond \mathfrak{x}_{2}\right) \otimes \varepsilon_{\mathrm{M}}\left(\mathfrak{x}_{1}^{\prime}\right) \varepsilon_{\mathrm{M}}\left(\mathfrak{x}_{2}^{\prime}\right) 1+\lambda\left(\mathfrak{x}_{1} \diamond \mathfrak{x}_{2}\right) \otimes P_{A}\left(\mathfrak{x}_{1}^{\prime}\right) \varepsilon_{\mathrm{M}}\left(\mathfrak{x}_{2}^{\prime}\right) \\
& -\lambda\left(P_{A}\left(\mathfrak{x}_{1} \diamond \mathfrak{x}_{2}\right)+\lambda \mathfrak{x}_{1} \diamond \mathfrak{x}_{2}\right) \otimes \varepsilon_{\mathrm{M}}\left(\mathfrak{x}_{1}^{\prime}\right) \varepsilon_{\mathrm{M}}\left(\mathfrak{x}_{2}^{\prime}\right) 1+\left(\mathfrak{x}_{1} \diamond \mathfrak{x}_{2}\right) \otimes P_{A}\left(\mathfrak{x}_{1}^{\prime} P_{A}\left(\mathfrak{x}_{2}^{\prime}\right)\right) \\
& +\left(P_{A}\left(P_{A}\left(\mathfrak{x}_{1}\right) \diamond \mathfrak{x}_{2}\right)+\lambda P_{A}\left(\mathfrak{x}_{1}\right) \diamond \mathfrak{x}_{2}\right) \otimes \varepsilon_{\mathrm{M}}\left(\mathfrak{x}_{1}^{\prime}\right) \varepsilon_{\mathrm{M}}\left(\mathfrak{x}_{2}^{\prime}\right) 1+\left(P_{A}\left(\mathfrak{x}_{1}\right) \diamond \mathfrak{x}_{2}\right) \otimes \varepsilon_{\mathrm{M}}\left(\mathfrak{x}_{1}^{\prime}\right) P_{A}\left(\mathfrak{x}_{2}^{\prime}\right) \\
& +\lambda\left(P_{A}\left(\mathfrak{x}_{1} \diamond \mathfrak{x}_{2}\right)+\lambda \mathfrak{x}_{1} \diamond \mathfrak{x}_{2}\right) \otimes \varepsilon_{\mathrm{M}}\left(\mathfrak{x}_{1}^{\prime}\right) \varepsilon_{\mathrm{M}}\left(\mathfrak{x}_{2}^{\prime}\right) 1+\lambda\left(\mathfrak{x}_{1} \diamond \mathfrak{x}_{2}\right) \otimes \varepsilon_{\mathrm{M}}\left(\mathfrak{x}_{1}^{\prime}\right) P_{A}\left(\mathfrak{x}_{2}^{\prime}\right) \\
& -\lambda\left(P_{A}\left(\mathfrak{x}_{1} \diamond \mathfrak{x}_{2}\right)+\lambda \mathfrak{x}_{1} \diamond \mathfrak{x}_{2}\right) \otimes \varepsilon_{\mathrm{M}}\left(\mathfrak{x}_{1}^{\prime}\right) \varepsilon_{\mathrm{M}}\left(\mathfrak{x}_{2}^{\prime}\right) 1+\left(\mathfrak{x}_{1} \diamond \mathfrak{x}_{2}\right) \otimes P_{A}\left(P_{A}\left(\mathfrak{x}_{1}^{\prime}\right) \mathfrak{x}_{2}^{\prime}\right) \\
& -\lambda^{2}\left(\mathfrak{x}_{1} \diamond \mathfrak{x}_{2}\right) \otimes\left(\mathfrak{x}_{1}^{\prime} \diamond \mathfrak{x}_{2}^{\prime}\right) \quad \text { (Using Eq. (13) in the fifth and eleventh terms) }
\end{aligned}
$$




$$
\begin{aligned}
= & \left(P_{A}\left(\mathfrak{x}_{1} \diamond P_{A}\left(\mathfrak{x}_{2}\right)\right)+\lambda \mathfrak{x}_{1} \diamond P_{A}\left(\mathfrak{x}_{2}\right)+P_{A}\left(P_{A}\left(\mathfrak{x}_{1}\right) \diamond \mathfrak{x}_{2}\right)+\lambda P_{A}\left(\mathfrak{x}_{1}\right) \diamond \mathfrak{x}_{2}\right) \otimes \varepsilon_{\mathrm{M}}\left(\mathfrak{x}_{1}^{\prime}\right) \varepsilon_{\mathrm{M}}\left(\mathfrak{x}_{2}^{\prime}\right) 1 \\
& +\left(\mathfrak{x}_{1} \diamond P_{A}\left(\mathfrak{x}_{2}\right)\right) \otimes P_{A}\left(\mathfrak{x}_{1}^{\prime}\right) \varepsilon_{\mathrm{M}}\left(\mathfrak{x}_{2}^{\prime}\right)+\lambda\left(\mathfrak{x}_{1} \diamond \mathfrak{x}_{2}\right) \otimes P_{A}\left(\mathfrak{x}_{1}^{\prime}\right) \varepsilon_{\mathrm{M}}\left(\mathfrak{x}_{2}^{\prime}\right)+\left(\mathfrak{x}_{1} \diamond \mathfrak{x}_{2}\right) \otimes P_{A}\left(\mathfrak{x}_{1}^{\prime} P_{A}\left(\mathfrak{x}_{2}^{\prime}\right)\right) \\
& +\left(P_{A}\left(\mathfrak{x}_{1}\right) \diamond \mathfrak{x}_{2}\right) \otimes \varepsilon_{\mathrm{M}}\left(\mathfrak{x}_{1}^{\prime}\right) P_{A}\left(\mathfrak{x}_{2}^{\prime}\right)+\lambda\left(\mathfrak{x}_{1} \diamond \mathfrak{x}_{2}\right) \otimes \varepsilon_{\mathrm{M}}\left(\mathfrak{x}_{1}^{\prime}\right) P_{A}\left(\mathfrak{x}_{2}^{\prime}\right)+\left(\mathfrak{x}_{1} \diamond \mathfrak{x}_{2}\right) \otimes P_{A}\left(P_{A}\left(\mathfrak{x}_{1}^{\prime}\right) \mathfrak{x}_{2}^{\prime}\right) \\
& -\lambda^{2}\left(\mathfrak{x}_{1} \diamond \mathfrak{x}_{2}\right) \otimes\left(\mathfrak{x}_{1}^{\prime} \diamond \mathfrak{x}_{2}^{\prime}\right) .
\end{aligned}
$$

This completes the proof.

With a similar argument, we can obtain

Lemma 3.3. Let $\lambda$ be a given element of $\mathbf{k}$. Define the linear map

$$
\tilde{Q}: F_{-\lambda^{2}}(A) \otimes F_{-\lambda^{2}}(A) \otimes F_{-\lambda^{2}}(A) \rightarrow F_{-\lambda^{2}}(A) \otimes F_{-\lambda^{2}}(A) \otimes F_{-\lambda^{2}}(A)
$$

by taking

$$
\begin{aligned}
\tilde{Q}\left(\mathfrak{x} \otimes \mathfrak{x}^{\prime} \otimes \mathfrak{x}^{\prime \prime}\right):= & \left(P_{A}(\mathfrak{x})+\lambda \mathfrak{x}\right) \otimes \varepsilon_{\mathrm{M}}\left(\mathfrak{x}^{\prime}\right) 1 \otimes \varepsilon_{\mathrm{M}}\left(\mathfrak{x}^{\prime \prime}\right) 1+\mathfrak{x} \otimes\left(P_{A}\left(\mathfrak{x}^{\prime}\right)+\lambda \mathfrak{x}^{\prime}\right) \otimes \varepsilon_{\mathrm{M}}\left(\mathfrak{x}^{\prime \prime}\right) 1 \\
& +\mathfrak{x} \otimes \mathfrak{x}^{\prime} \otimes P_{A}\left(\mathfrak{x}^{\prime \prime}\right) \text { for } \mathfrak{x}, \mathfrak{x}^{\prime} \in F_{-\lambda^{2}}(A) .
\end{aligned}
$$

Then $\tilde{Q}$ is a modified Rota-Baxter operator of weight $-\lambda^{2}$ on $F_{-\lambda^{2}}(A) \otimes F_{-\lambda^{2}}(A) \otimes$ $F_{-\lambda^{2}}(A)$.

Now we are ready for our main result of this subsection. Recall $\varepsilon_{\mathrm{M}}: F_{-\lambda^{2}}(A) \rightarrow$ $\mathbf{k}$ is an algebra homomorphism given in Lemma 3.1. Let $j_{A}: A \rightarrow F_{\kappa}(A)$ be the natural embedding. By Theorem 2.6 (c) and Lemma 3.2, there is a (unique) modified Rota-Baxter algebra morphism

$$
\Delta_{M}: F_{-\lambda^{2}}(A) \rightarrow F_{-\lambda^{2}}(A) \otimes F_{-\lambda^{2}}(A)
$$

such that $\Delta_{M} \circ j_{A}=\Delta_{A}$.

Theorem 3.4. Let $A$ be a bialgebra and $\lambda \in \mathbf{k}$. Then the quintuple $\left(F_{-\lambda^{2}}(A), \diamond, 1, \Delta_{\mathrm{M}}, \varepsilon_{\mathrm{M}}\right)$ is a bialgebra.

Proof. It suffices to prove the counity of $\varepsilon_{\mathrm{M}}$ and coassociativity of $\Delta_{\mathrm{M}}$. For the former, denote by

$$
\phi:=\left(\varepsilon_{\mathrm{M}} \otimes \mathrm{id}\right) \Delta_{M}: F_{-\lambda^{2}}(A) \rightarrow F_{-\lambda^{2}}(A)
$$

Then $\phi$ is an algebra homomorphism, since $\varepsilon_{\mathrm{M}}$ and $\Delta_{M}$ are algebra homomorphisms. Further it is a modified Rota-Baxter algebra morphism. Indeed, for any $\mathfrak{x} \in F_{-\lambda^{2}}(A)$, $\phi \circ P_{A}(\mathfrak{x})=\left(\left(\varepsilon_{\mathrm{M}} \otimes \mathrm{id}\right) \Delta_{M}\right) P_{A}(\mathfrak{x})=\left(\varepsilon_{\mathrm{M}} \otimes \mathrm{id}\right)\left(\Delta_{M} P_{A}\right)(\mathfrak{x})$ 


$$
\begin{aligned}
& =\left(\varepsilon_{\mathrm{M}} \otimes \mathrm{id}\right)\left(Q \Delta_{M}\right)(\mathfrak{x}) \quad\left(\text { by } \Delta_{M} \text { being an modified Rota-Baxter algebra morphism }\right) \\
& =\left(\varepsilon_{\mathrm{M}} \otimes \mathrm{id}\right) Q\left(\sum_{(\mathfrak{x})} \mathfrak{x}_{(1)} \otimes \mathfrak{x}_{(2)}\right) \quad \text { (by Sweedler's notation) } \\
& =\sum_{(\mathfrak{x})}\left(\varepsilon_{\mathrm{M}} \otimes \mathrm{id}\right)\left(\left(P_{A}\left(\mathfrak{x}_{(1)}\right)+\lambda \mathfrak{x}_{(1)}\right) \otimes \varepsilon_{\mathrm{M}}\left(\mathfrak{x}_{(2)}\right) 1+\mathfrak{x}_{(1)} \otimes P_{A}\left(\mathfrak{x}_{(2)}\right)\right) \quad \text { (by Eq. (14)) } \\
& =\sum_{(\mathfrak{x})}\left(\varepsilon_{\mathrm{M}}\left(P_{A}\left(\mathfrak{x}_{(1)}\right)\right) \otimes \varepsilon_{\mathrm{M}}\left(\mathfrak{x}_{(2)}\right) 1+\lambda \varepsilon_{\mathrm{M}}\left(\mathfrak{x}_{(1)}\right) \otimes \varepsilon_{\mathrm{M}}\left(\mathfrak{x}_{(2)}\right) 1+\varepsilon_{\mathrm{M}}\left(\mathfrak{x}_{(1)}\right) \otimes P_{A}\left(\mathfrak{x}_{(2)}\right)\right) \\
& =\sum_{(\mathfrak{x})}\left(-\lambda \varepsilon_{\mathrm{M}}\left(\mathfrak{x}_{(1)}\right) \otimes \varepsilon_{\mathrm{M}}\left(\mathfrak{x}_{(2)}\right) 1+\lambda \varepsilon_{\mathrm{M}}\left(\mathfrak{x}_{(1)}\right) \otimes \varepsilon_{\mathrm{M}}\left(\mathfrak{x}_{(2)}\right) 1+\varepsilon_{\mathrm{M}}\left(\mathfrak{x}_{(1)}\right) \otimes P_{A}\left(\mathfrak{x}_{(2)}\right)\right) \\
& =\sum_{(\mathfrak{x})} \varepsilon_{\mathrm{M}}\left(\mathfrak{x}_{(1)}\right) \otimes P_{A}\left(\mathfrak{x}_{(2)}\right)=\sum_{(\mathfrak{x})} P_{A}\left(\varepsilon_{\mathrm{M}}\left(\mathfrak{x}_{(1)}\right) \mathfrak{x}_{(2)}\right)=P_{A}\left(\left(\varepsilon_{\mathrm{M}} \otimes \mathrm{id}\right) \Delta_{M}(\mathfrak{x})\right) \\
& =P_{A} \circ \phi(\mathfrak{x}) .
\end{aligned}
$$

By unicity in the universal property of $F_{-\lambda^{2}}(A)$, we have

$$
\left(\varepsilon_{\mathrm{M}} \otimes \mathrm{id}\right) \Delta_{M}=\phi=\mathrm{id}_{F_{-\lambda^{2}}(A)}
$$

and so $\varepsilon_{\mathrm{M}}$ is a left counit. By symmetry, we can prove $\varepsilon_{\mathrm{M}}$ is also a right counit.

Moreover, both $\left(\Delta_{M} \otimes \mathrm{id}\right) \Delta_{M}$ and $\left(\mathrm{id} \otimes \Delta_{M}\right) \Delta_{M}$ are modified Rota-Baxter algebra morphisms from $F_{-\lambda^{2}}(A)$ to $F_{-\lambda^{2}}(A) \otimes F_{-\lambda^{2}}(A) \otimes F_{-\lambda^{2}}(A)$, which is equipped with the modified Rota-Baxter operator $\tilde{Q}$ of weight $-\lambda^{2}$ given in Lemma 3.3. As they coincide on $A$

$$
\left.\left(\Delta_{M} \otimes \mathrm{id}\right) \Delta_{M}\right|_{A}=\left(\Delta_{A} \otimes \mathrm{id}\right) \Delta_{A}=\left(\mathrm{id} \otimes \Delta_{A}\right) \Delta_{A}=\left.\left(\mathrm{id} \otimes \Delta_{M}\right) \Delta_{M}\right|_{A},
$$

they are equal and so $\Delta_{M}$ is coassociative. Here $\Delta_{A}$ is the coproduct on $A$. Thus the quintuple $\left(F_{-\lambda^{2}}(A), \diamond, 1, \Delta_{\mathrm{M}}, \varepsilon_{\mathrm{M}}\right)$ is a bialgebra.

Remark 3.5. For any $\mathfrak{x} \in F_{-\lambda^{2}}(A)$, we have

$$
\begin{aligned}
\Delta_{M} \circ P_{A}(\mathfrak{x}) & =Q \circ \Delta_{M}(\mathfrak{x}) \quad\left(\text { by } \Delta_{M} \text { being a modified Rota-Baxter algebra morphism }\right) \\
& \left.=Q\left(\sum_{(\mathfrak{x})} \mathfrak{x}_{(1)} \otimes \mathfrak{x}_{(2)}\right) \quad \text { (by Sweedler's notation }\right) \\
& =\sum_{(\mathfrak{x})}\left(\left(P_{A}\left(\mathfrak{x}_{(1)}\right)+\lambda \mathfrak{x}_{(1)}\right) \otimes \varepsilon_{\mathrm{M}}\left(\mathfrak{x}_{(2)}\right) 1+\mathfrak{x}_{(1)} \otimes P_{A}\left(\mathfrak{x}_{(2)}\right)\right) \quad(\text { by Eq. (14) }) \\
& =\sum_{(\mathfrak{x})}\left(P_{A}\left(\mathfrak{x}_{(1)}\right) \otimes \varepsilon_{\mathrm{M}}\left(\mathfrak{x}_{(2)}\right) 1+\lambda \mathfrak{x}_{(1)} \otimes \varepsilon_{\mathrm{M}}\left(\mathfrak{x}_{(2)}\right) 1+\mathfrak{x}_{(1)} \otimes P_{A}\left(\mathfrak{x}_{(2)}\right)\right)
\end{aligned}
$$




$$
\begin{aligned}
& =\sum_{(\mathfrak{x})}\left(P_{A}\left(\mathfrak{x}_{(1)} \varepsilon_{\mathrm{M}}\left(\mathfrak{x}_{(2)}\right)\right) \otimes 1+\lambda \mathfrak{x}_{(1)} \varepsilon_{\mathrm{M}}\left(\mathfrak{x}_{(2)}\right) \otimes 1+\left(\mathrm{id} \otimes P_{A}\right)\left(\mathfrak{x}_{(1)} \otimes \mathfrak{x}_{(2)}\right)\right) \\
& =P_{A}(\mathfrak{x}) \otimes 1+\lambda \mathfrak{x} \otimes 1+\left(\mathrm{id} \otimes P_{A}\right) \Delta_{M}(\mathfrak{x}) \quad\left(\text { by } \varepsilon_{M} \text { being the counit }\right) .
\end{aligned}
$$

In other words,

$$
\Delta_{M} P_{A}=P_{A} \otimes 1+\left(\mathrm{id} \otimes P_{A}\right) \Delta_{M}+\lambda \mathrm{id} \otimes 1,
$$

which is analogue to the 1-cocycle condition in the well-known Connes-Kreimer Hopf algebra on rooted trees [11].

3.2. The Hopf algebraic structure. In this last part of the paper we show that if we start with $A$ being a connected filtered bialgebra and $\lambda \in \mathbf{k}$, then the bialgebra $F_{-\lambda^{2}}(A)$ also has a connected filtration and hence is a Hopf algebra.

Definition 3.6. A bialgebra $(A, m, \mu, \Delta, \varepsilon)$ is called filtered if it has an increasing filtration $A_{n}, n \geq 0$, such that

$$
A=\cup_{n \geq 0} A_{n}, A_{p} A_{q} \subseteq A_{p+q} \text { and } \Delta\left(A_{n}\right) \subseteq \sum_{p+q=n} A_{p} \otimes A_{q} \text { for } p, q, n \geq 0 .
$$

A filtered bialgebra $A$ is called connected if $A_{0}=\operatorname{im} \mu$ and $A=A_{0} \oplus \operatorname{ker} \varepsilon$.

The following result is well-known.

Lemma 3.7. [23] A connected filtered bialgebra is a Hopf algebra.

Our discussion in this section will be based on the following condition.

Definition 3.8. A k-basis $X$ of a connected filtered bialgebra $A=\cup_{n \geq 0} A_{n}$ is called a filtered basis of $A$ if there is an increasing filtration $X=\cup_{n \geq 0} X_{n}$ such that

$$
A_{n}=\mathbf{k} X_{n}, X \backslash\{1\} \subseteq \operatorname{ker} \varepsilon, X_{0}=\{1\} .
$$

Here 1 is the identity of $A$. Elements $x \in X_{n} \backslash X_{n-1}$ are said to have degree $n$, denoted by $\operatorname{deg}_{A}(x)=n$.

Let $A$ be a connected filtered bialgebra with a filtered basis $X$. Recall that $\mathfrak{X}_{\infty}$ constructed in Subsection 2.1 is a $\mathbf{k}$-basis of the free modified Rota-Baxter algebra $F_{-\lambda^{2}}(A)$. We now define the degree $\operatorname{deg}(\mathfrak{x})$ for $\mathfrak{x} \in \mathfrak{X}_{\infty}$ by induction on $\operatorname{dep}(\mathfrak{x})$. For the initial step of $\operatorname{dep}(\mathfrak{x})=0$, we get $\mathfrak{x} \in X \subseteq A$ and define

$$
\operatorname{deg}(\mathfrak{x}):=\operatorname{deg}_{A}(\mathfrak{x}) .
$$

For the inductive step of $\operatorname{dep}(\mathfrak{x}) \geq 1$, if $\operatorname{bre}(\mathfrak{x})=1$, then $\mathfrak{x}=\lfloor\overline{\mathfrak{x}}\rfloor$ and we define

$$
\operatorname{deg}(\mathfrak{x}):=\operatorname{deg}(\overline{\mathfrak{x}})+1
$$


if $\operatorname{bre}(\mathfrak{x}) \geq 2$, then write $\mathfrak{x}=\mathfrak{x}_{1} \cdots \mathfrak{x}_{b}$ in the standard decomposition and define

$$
\operatorname{deg}(\mathfrak{x}):=\sum_{i=1}^{b} \operatorname{deg}\left(\mathfrak{x}_{i}\right)
$$

where each $\operatorname{deg}\left(\mathfrak{x}_{i}\right)$ is defined either in Eq. (17) or in Eq. (18) by the induction hypothesis.

Remark 3.9. For later applications, we also use the notion $\operatorname{deg}(c \mathfrak{x})=\operatorname{deg}(\mathfrak{x})$ for $c \in \mathbf{k} \backslash\{0\}$.

Denote

$$
\mathcal{H}:=F_{-\lambda^{2}}(A) \text { and } \mathcal{H}_{n}:=\mathbf{k}\left\{\mathfrak{x} \in \mathfrak{X}_{\infty} \mid \operatorname{deg}(\mathfrak{x}) \leq n\right\} \text { for } n \geq 0 \text {. }
$$

Then

$$
\mathcal{H}=\bigcup_{n \geq 0} \mathcal{H}_{n}, \mathcal{H}_{0}=\mathbf{k}, \mathcal{H}=\mathbf{k} 1 \oplus \operatorname{ker} \varepsilon_{\mathrm{M}} \text { and } P_{A}\left(\mathcal{H}_{n}\right) \subseteq \mathcal{H}_{n+1}
$$

Now we are going to prove that $\mathcal{H}$ is a filtered bialgebra, beginning with the compatibility of the multiplication with the filtration.

Lemma 3.10. For $p, q \geq 0$, we have

$$
\mathcal{H}_{p} \diamond \mathcal{H}_{q} \subseteq \mathcal{H}_{p+q}
$$

Proof. Let $\mathfrak{x} \in \mathcal{H}_{p}$ and $\mathfrak{x}^{\prime} \in \mathcal{H}_{q}$ be two basis elements in $\mathfrak{X}_{\infty}$. Then

$$
\operatorname{deg}(\mathfrak{x}) \leq p \text { and } \operatorname{deg}\left(\mathfrak{x}^{\prime}\right) \leq q .
$$

We now verify Eq. (22) by induction on the sum $s:=p+q \geq 0$. When $s=0$, then $p=q=0$. By Eq. (21), we obtain that $\mathfrak{x}=\mathfrak{x}^{\prime}=1$ and so $\mathfrak{x} \diamond \mathfrak{x}^{\prime}=1 \in \mathcal{H}_{0}$. This finishes the initial step.

Given an $s \geq 0$, assume that Eq. (22) holds for $\mathfrak{x}, \mathfrak{x}^{\prime}$ with $p+q=s$ and consider case $p+q=s+1$. If $\mathfrak{x}=1$ or $\mathfrak{x}^{\prime}=1$, without loss of generality, letting $\mathfrak{x}=1$, then $p=0$ and

$$
\mathfrak{x} \diamond \mathfrak{x}^{\prime}=\mathfrak{x}^{\prime} \in \mathcal{H}_{q}=\mathcal{H}_{p+q} .
$$

So we may suppose $\mathfrak{x}, \mathfrak{x}^{\prime} \neq 1$. Write

$$
\mathfrak{x}=\mathfrak{x}_{1} \cdots \mathfrak{x}_{b} \text { and } \mathfrak{x}^{\prime}:=\mathfrak{x}_{1}^{\prime} \cdots \mathfrak{x}_{b^{\prime}}^{\prime} \text { with } b, b^{\prime} \geq 1
$$


in their standard decompositions. Under this condition, we proceed to prove Eq. (22) by induction on the sum $t:=b+b^{\prime} \geq 2$. When $t=2$, then $b=b^{\prime}=1$. If $\mathfrak{x} \in X \subseteq A$ or $\mathfrak{x}^{\prime} \in X \subseteq A$, then by Eq. (19),

$$
\mathfrak{x} \diamond \mathfrak{x}^{\prime}=\mathfrak{x x}^{\prime} \in \mathcal{H}_{\operatorname{deg}(\mathfrak{x})+\operatorname{deg}\left(\mathfrak{x}^{\prime}\right)} \subseteq \mathcal{H}_{p+q} .
$$

It remains to check the outstanding case of

$$
\mathfrak{x}:=\mathfrak{x}_{1}=P_{A}\left(\overline{\mathfrak{x}}_{1}\right) \text { and } \mathfrak{x}^{\prime}:=\mathfrak{x}_{1}^{\prime}=P_{A}\left(\overline{\mathfrak{x}}_{1}^{\prime}\right),
$$

where

$$
\overline{\mathfrak{x}}_{1}, \overline{\mathfrak{x}}_{1}^{\prime} \in \mathfrak{X}_{\infty} \text { and } \operatorname{deg}\left(\mathfrak{x}_{1}\right)+\operatorname{deg}\left(\mathfrak{x}_{1}^{\prime}\right) \leq s+1
$$

Then

$$
\begin{aligned}
& \operatorname{deg}\left(\mathfrak{x}_{1}\right)+\operatorname{deg}\left(\overline{\mathfrak{x}}_{1}^{\prime}\right)=\operatorname{deg}\left(\mathfrak{x}_{1}\right)+\operatorname{deg}\left(\mathfrak{x}_{1}^{\prime}\right)-1 \leq s, \\
& \operatorname{deg}\left(\overline{\mathfrak{x}}_{1}\right)+\operatorname{deg}\left(\mathfrak{x}_{1}^{\prime}\right)=\operatorname{deg}\left(\mathfrak{x}_{1}\right)-1+\operatorname{deg}\left(\mathfrak{x}_{1}^{\prime}\right) \leq s, \\
& \operatorname{deg}\left(\overline{\mathfrak{x}}_{1}\right)+\operatorname{deg}\left(\overline{\mathfrak{x}_{1}^{\prime}}\right)=\operatorname{deg}\left(\mathfrak{x}_{1}\right)-1+\operatorname{deg}\left(\mathfrak{x}_{1}^{\prime}\right)-1 \leq s-1 .
\end{aligned}
$$

By the induction hypothesis on $s$, we have

$$
\mathfrak{x}_{1} \diamond \overline{\mathfrak{x}}_{1}^{\prime}, \overline{\mathfrak{x}}_{1} \diamond \mathfrak{x}_{1}^{\prime} \in \mathcal{H}_{s} \text { and } \overline{\mathfrak{x}_{1}} \diamond \overline{\mathfrak{x}}_{1}^{\prime} \in \mathcal{H}_{s-1}
$$

which implies from Eq. (21) that

$$
P_{A}\left(\mathfrak{x}_{1} \diamond \overline{\mathfrak{x}}_{1}^{\prime}\right), P_{A}\left(\overline{\mathfrak{x}}_{1} \diamond \mathfrak{x}_{1}^{\prime}\right) \in \mathcal{H}_{s+1} .
$$

Hence by Eq. (7),

$$
\mathfrak{x}_{1} \diamond \mathfrak{x}_{1}^{\prime}=P_{A}\left(\mathfrak{x}_{1} \diamond \overline{\mathfrak{x}}_{1}^{\prime}\right)+P_{A}\left(\overline{\mathfrak{x}}_{1} \diamond \mathfrak{x}_{1}^{\prime}\right)-\lambda^{2} \overline{\mathfrak{x}}_{1} \diamond \overline{\mathfrak{x}_{1}^{\prime}} \in \mathcal{H}_{s+1} .
$$

Assume that Eq. (22) holds for $b+b^{\prime}=t \geq 2$ and $p+q=s+1$ and consider the case when $b+b^{\prime}=t+1 \geq 3$ and $p+q=s+1$. So either $\mathfrak{x}$ or $\mathfrak{x}^{\prime}$ has breadth greater than or equal to 2 , giving us three cases to consider:

Case 1. $\operatorname{bre}(\mathfrak{x}) \geq 2$. Let $\mathfrak{x}:=\mathfrak{x}_{1,1} \mathfrak{x}_{1,2}$, where $\mathfrak{x}_{1,1}, \mathfrak{x}_{1,2} \in \mathfrak{X}_{\infty}$ with breadths $\operatorname{bre}\left(\mathfrak{x}_{1,1}\right)$, bre $\left(\mathfrak{x}_{1,2}\right) \geq 1$ respectively. By Eq. (19), we obtain $\operatorname{deg}(\mathfrak{x})=\operatorname{deg}\left(\mathfrak{x}_{1,1}\right)+$ $\operatorname{deg}\left(\mathfrak{x}_{1,2}\right)$. From Eq. (8),

$$
\mathfrak{x} \diamond \mathfrak{x}^{\prime}=\left(\mathfrak{x}_{1,1} \mathfrak{x}_{1,2}\right) \diamond \mathfrak{x}^{\prime}=\mathfrak{x}_{1,1}\left(\mathfrak{x}_{1,2} \diamond \mathfrak{x}^{\prime}\right) .
$$

By the induction on $t$, we have

$$
\mathfrak{x}_{1,2} \diamond \mathfrak{x}^{\prime} \in \mathcal{H}_{\operatorname{deg}\left(\mathfrak{x}_{1,2}\right)+\operatorname{deg}\left(\mathfrak{x}^{\prime}\right)},
$$

whence by Eq. (19),

$$
\mathfrak{x} \diamond \mathfrak{x}^{\prime}=\mathfrak{x}_{1,1}\left(\mathfrak{x}_{1,2} \diamond \mathfrak{x}^{\prime}\right) \in \mathcal{H}_{\operatorname{deg}\left(\mathfrak{x}_{1,1}\right)+\operatorname{deg}\left(\mathfrak{x}_{1,2}\right)+\operatorname{deg}\left(\mathfrak{x}^{\prime}\right)}=\mathcal{H}_{\operatorname{deg}(\mathfrak{x})+\operatorname{deg}\left(\mathfrak{x}^{\prime}\right)} .
$$


Case 2. bre $\left(\mathfrak{x}^{\prime}\right) \geq 2$. The proof of this case is similar to Case 1 .

Case 3. bre $(\mathfrak{x}) \geq 2$ and bre $\left(\mathfrak{x}^{\prime}\right) \geq 2$. Let $\mathfrak{x}:=\mathfrak{x}_{1,1} \mathfrak{x}_{1,2}$ and $\mathfrak{x}^{\prime}:=\mathfrak{x}_{1,1}^{\prime} \mathfrak{x}_{1,2}^{\prime}$, where $\mathfrak{x}_{1,1}, \mathfrak{x}_{1,2}, \mathfrak{x}_{1,1}^{\prime}, \mathfrak{x}_{1,2}^{\prime} \in \mathfrak{X}_{\infty}$ with breadths bre $\left(\mathfrak{x}_{1,1}\right)$, bre $\left(\mathfrak{x}_{1,2}\right)$, bre $\left(\mathfrak{x}_{1,1}^{\prime}\right)$, bre $\left(\mathfrak{x}_{1,2}^{\prime}\right) \geq 1$ respectively. By Eq. (19), we obtain

$$
\operatorname{deg}(\mathfrak{x})=\operatorname{deg}\left(\mathfrak{x}_{1,1}\right)+\operatorname{deg}\left(\mathfrak{x}_{1,2}\right) \text { and } \operatorname{deg}\left(\mathfrak{x}^{\prime}\right)=\operatorname{deg}\left(\mathfrak{x}_{1,1}^{\prime}\right)+\operatorname{deg}\left(\mathfrak{x}_{1,2}^{\prime}\right) .
$$

Thus by Eq. (8),

$$
\mathfrak{x} \diamond \mathfrak{x}^{\prime}=\left(\mathfrak{x}_{1,1} \mathfrak{x}_{1,2}\right) \diamond\left(\mathfrak{x}_{1,1}^{\prime} \mathfrak{x}_{1,2}^{\prime}\right)=\mathfrak{x}_{1,1}\left(\mathfrak{x}_{1,2} \diamond \mathfrak{x}_{1,1}^{\prime}\right) \mathfrak{x}_{1,2}^{\prime} .
$$

By the induction on $t$, we have

$$
\mathfrak{x}_{1,2} \diamond \mathfrak{x}_{1,1}^{\prime} \in \mathcal{H}_{\operatorname{deg}\left(\mathfrak{x}_{1,2}\right)+\operatorname{deg}\left(\mathfrak{x}_{1,1}^{\prime}\right)} .
$$

With a similar argument to Case 1 . we get

$$
\mathfrak{x} \diamond \mathfrak{x}^{\prime}=\mathfrak{x}_{1,1}\left(\mathfrak{x}_{1,2} \diamond \mathfrak{x}_{1,1}^{\prime}\right) \mathfrak{x}_{1,2}^{\prime} \in \mathcal{H}_{\operatorname{deg}(\mathfrak{x})+\operatorname{deg}\left(\mathfrak{x}^{\prime}\right)} .
$$

This finishes the proof.

For the compatibility of the coproduct with the filtration, we have

Lemma 3.11. For $n \geq 0$, we have

$$
\Delta_{\mathrm{M}}\left(\mathcal{H}_{n}\right) \subseteq \sum_{p+q=n} \mathcal{H}_{p} \otimes \mathcal{H}_{q} .
$$

Proof. We verify Eq. (23) by showing

Claim 3.12. For any $\mathfrak{x} \in \mathfrak{X}_{\infty}$, we have

$$
\Delta_{\mathrm{M}}(\mathfrak{x})=\sum_{(\mathfrak{x})} \mathfrak{x}_{(1)} \otimes \mathfrak{x}_{(2)},
$$

where $\mathfrak{x}_{(1)}$ and $\mathfrak{x}_{(2)}$ are non-zero linear multiples of elements of $\mathfrak{X}_{\infty}$ with $\operatorname{deg}\left(\mathfrak{x}_{(1)}\right)+$ $\operatorname{deg}\left(\mathfrak{x}_{(2)}\right) \leq \operatorname{deg}(\mathfrak{x})$. Here we have adapted the notation in Remark 3.9.

To prove this claim we proceed by induction on $\operatorname{deg}(\mathfrak{x}) \geq 0$. For the initial step of $\operatorname{deg}(\mathfrak{x})=0$, we get $\mathfrak{x}=1$ and the result holds. Assume that Claim (3.12) holds for $\mathfrak{x} \in \mathcal{H}_{k}$ and consider $\mathfrak{x} \in \mathcal{H}_{k+1}$ for some $k \geq 0$.

In this case, we prove Claim (3.12) by induction on the breadth $b:=\operatorname{bre}(\mathfrak{x}) \geq 1$. If $b=1$, we have $\mathfrak{x} \in X \subseteq A$ or $\mathfrak{x}=P_{A}(\overline{\mathfrak{x}})$ for some $\overline{\mathfrak{x}} \in \mathfrak{X}_{\infty}$. For the former, Claim (3.12) holds since $\Delta_{\mathrm{M}}$ is given by $\Delta_{A}$ and $A$ is a connected filtered bialgebra 
by our hypothesis. For the latter, applying the induction hypothesis on $n$, we can write

$$
\Delta_{\mathrm{M}}(\overline{\mathfrak{x}})=\sum_{(\overline{\mathfrak{x}})} \overline{\mathfrak{x}}_{(1)} \otimes \overline{\mathfrak{x}}_{(2)},
$$

where $\operatorname{deg}\left(\overline{\mathfrak{x}}_{(1)}\right)+\operatorname{deg}\left(\overline{\mathfrak{x}}_{(2)}\right) \leq \operatorname{deg}(\overline{\mathfrak{x}})=k$, with the notion in Remark 3.9. By Eq. (16), we have

$$
\begin{aligned}
\Delta_{\mathrm{M}}(\mathfrak{x})=\Delta_{\mathrm{M}}\left(P_{A}(\overline{\mathfrak{x}})\right) & =\mathfrak{x} \otimes 1+\left(\mathrm{id} \otimes P_{A}\right) \Delta_{\mathrm{M}}(\overline{\mathfrak{x}})+\lambda \overline{\mathfrak{x}} \otimes 1 \\
& =\mathfrak{x} \otimes 1+\sum_{(\overline{\mathfrak{x}})}\left(\overline{\mathfrak{x}}_{(1)} \otimes P_{A}\left(\overline{\mathfrak{x}}_{(2)}\right)+\lambda \overline{\mathfrak{x}} \otimes 1 .\right.
\end{aligned}
$$

By Eq. (20), it is sufficient to show that the sum of degrees of tensor factors in each summand is less than or equal to $k+1$, which follows from

$$
\begin{aligned}
& \operatorname{deg}(\mathfrak{x})+\operatorname{deg}(1)=\operatorname{deg}(\mathfrak{x}) \leq k+1, \operatorname{deg}(\overline{\mathfrak{x}})+\operatorname{deg}(1)=\operatorname{deg}(\overline{\mathfrak{x}}) \leq k, \\
& \operatorname{deg}\left(\overline{\mathfrak{x}}_{(1)}\right)+\operatorname{deg}\left(P_{A}\left(\overline{\mathfrak{x}}_{(2)}\right)\right)=\operatorname{deg}\left(\overline{\mathfrak{x}}_{(1)}\right)+\operatorname{deg}\left(\overline{\mathfrak{x}}_{(2)}\right)+1 \leq k+1 .
\end{aligned}
$$

Assume that Claim (3.12) holds for $\mathfrak{x} \in \mathcal{H}_{k+1}$ with bre $(\mathfrak{x})=b$ and consider the case $\mathfrak{x} \in \mathcal{H}_{k+1}$ with $\operatorname{bre}(\mathfrak{x})=b+1 \geq 2$. Let $\mathfrak{x}=\mathfrak{x}_{1} \mathfrak{x}_{2}$, where $\mathfrak{x}_{1}, \mathfrak{x}_{2} \in \mathfrak{X}_{\infty}$ with $\operatorname{bre}\left(\mathfrak{x}_{1}\right)$, bre $\left(\mathfrak{x}_{2}\right) \geq 1$. From Eq. (19), we have

$$
\operatorname{deg}\left(\mathfrak{x}_{1}\right)+\operatorname{deg}\left(\mathfrak{x}_{2}\right)=\operatorname{deg}(\mathfrak{x}) \leq k+1 .
$$

Write

$$
\Delta_{\mathrm{M}}\left(\mathfrak{x}_{1}\right)=\sum_{\left(\mathfrak{x}_{1}\right)} \mathfrak{x}_{1(1)} \otimes \mathfrak{x}_{1(2)} \text { and } \Delta_{\mathrm{M}}\left(\mathfrak{x}_{2}\right)=\sum_{\left(\mathfrak{x}_{2}\right)} \mathfrak{x}_{2(1)} \otimes \mathfrak{x}_{2(2)} .
$$

By the induction hypothesis on $b$, we have

$$
\operatorname{deg}\left(\mathfrak{x}_{1(1)}\right)+\operatorname{deg}\left(\mathfrak{x}_{1(2)}\right) \leq \operatorname{deg}\left(\mathfrak{x}_{1}\right) \text { and } \operatorname{deg}\left(\mathfrak{x}_{2(1)}\right)+\operatorname{deg}\left(\mathfrak{x}_{2(2)}\right) \leq \operatorname{deg}\left(\mathfrak{x}_{2}\right) .
$$

So we have

$$
\begin{aligned}
\Delta_{\mathrm{M}}(\mathfrak{x}) & =\Delta_{\mathrm{M}}\left(\mathfrak{x}_{1} \mathfrak{x}_{2}\right)=\Delta_{\mathrm{M}}\left(\mathfrak{x}_{1} \diamond \mathfrak{x}_{2}\right)=\Delta_{\mathrm{M}}\left(\mathfrak{x}_{1}\right) \diamond \Delta_{\mathrm{M}}\left(\mathfrak{x}_{2}\right) \\
& =\left(\sum_{\left(\mathfrak{x}_{1}\right)} \mathfrak{x}_{1(1)} \otimes \mathfrak{x}_{1(2)}\right) \diamond\left(\sum_{\left(\mathfrak{x}_{2}\right)} \mathfrak{x}_{2(1)} \otimes \mathfrak{x}_{2(2)}\right) \\
& =\sum_{\left(\mathfrak{x}_{1}\right)} \sum_{\left(\mathfrak{x}_{2}\right)}\left(\mathfrak{x}_{1(1)} \diamond \mathfrak{x}_{2(1)}\right) \otimes\left(\mathfrak{x}_{1(2)} \diamond \mathfrak{x}_{2(2)}\right) .
\end{aligned}
$$

By Eq. (22),

$$
\mathfrak{x}_{1(1)} \diamond \mathfrak{x}_{2(1)} \in \mathcal{H}_{\operatorname{deg}\left(\mathfrak{x}_{1(1)}\right)+\operatorname{deg}\left(\mathfrak{x}_{2(1)}\right)} \text { and } \mathfrak{x}_{1(2)} \diamond \mathfrak{x}_{2(2)} \in \mathcal{H}_{\operatorname{deg}\left(\mathfrak{x}_{1(2)}\right)+\operatorname{deg}\left(\mathfrak{x}_{2(2)}\right)},
$$

which implies from Eqs. (20), (25) and (26) that Claim 3.12 holds.

We now arrive at our last main result. 
Theorem 3.13. Let $A=\cup_{n \geq 0} A_{n}$ be a connected filtered bialgebra with a filtered basis. Then $\mathcal{H}=F_{-\lambda^{2}}(A)$ is also a connected filtered bialgebra, and hence a Hopf algebra.

Proof. By Lemma 3.7, we just need to prove that $F_{-\lambda^{2}}(A)$ is a connected filtered bialgebra. This follows from Lemmas 3.10, 3.11 and Eq. (21).

Acknowledgement: The authors thank the referees for valuable suggestions and comments.

\section{References}

[1] M. Aguiar, On the associative analog of Lie bialgebras, J. Algebra, 244(2) (2001), 492-532.

[2] F. V. Atkinson, Some aspects of Baxter's functional equation, J. Math. Anal. Appl., 7 (1963), 1-30.

[3] C. Bai, O. Bellier, L. Guo and X. Ni, Splitting of operations, Manin products, and Rota-Baxter operators, Int. Math. Res. Not. IMRN, (2013) 3 (2013), 485524.

[4] C. Bai, L. Guo and X. Ni, Nonabelian generalized Lax pairs, the classical YangBaxter equation and PostLie algebras, Comm. Math. Phys., 297(2) (2010), 553-596.

[5] C. Bai, L. Guo and X. Ni, Generalizations of the classical Yang-Baxter equation and O-operators, J. Math. Phys., 52(6) (2011), 063515 (17 pp).

[6] C. Bai, L. Guo and X. Ni, O-operators on associative algebras and associative Yang-Baxter equations, Pacific J. Math., 256(2) (2012), 257-289.

[7] C. Bai, L. Guo and X. Ni, O-operators on associative algebras, associative Yang-Baxter equations and dendriform algebras, In "Quantized Algebra and Physics", Nankai Ser. Pure Appl. Math. Theoret. Phys., 8, World Sci. Publ., Hackensack, NJ, (2012), 10-51.

[8] G. Baxter, An analytic problem whose solution follows from a simple algebraic identity, Pacific J. Math., 10 (1960), 731-742.

[9] M. Bordemann, Generalized Lax pairs, the modified classical Yang-Baxter equation, and affine geometry of Lie groups, Comm. Math. Phys., 135(1) (1990), 201-216.

[10] P. Cartier, On the structure of free Baxter algebras, Advances in Math., 9 (1972), 253-265. 
[11] A. Connes and D. Kreimer, Hopf algebras, renormalization and noncommutative geometry, Comm. Math. Phys., 199(1) (1998), 203-242.

[12] K. Ebrihimi-Fard, Loday-type algebras and the Rota-Baxter relation, Lett. Math. Phys., 61(2) (2002), 139-147.

[13] K. Ebrahimi-Fard and L. Guo, Quasi-shuffles, mixable shuffles and Hopf algebras, J. Algebraic Combin., 24(1) (2006), 83-101.

[14] K. Ebrahimi-Fard and L. Guo, Rota-Baxter algebras and dendriform algebras, J. Pure Appl. Algebra, 212(2) (2008), 320-339.

[15] X. Gao, L. Guo and T. Zhang, Bialgebra and Hopf algebra structures on free Rota-Baxter algebra, arXiv:1604.03238.

[16] L. Guo, An Introduction to Rota-Baxter Algebra, Surveys of Modern Mathematics, 4, International Press, Somerville, MA; Higher Education Press, Beijing, 2012.

[17] L. Guo and W. Keigher, Baxter algebras and shuffle products, Adv. Math., 150(1) (2000), 117-149.

[18] L. Guo and W. Y. Sit Enumeration and generating functions of Rota-Baxter words, Math. Comput. Sci., 4(2-3) (2010), 313-337.

[19] R. Jian and J. Zhang, Rota-Baxter coalgebras, arXiv:1409.3052.

[20] Y. Kosmann-Schwarzbach, Lie bialgebras, Poisson Lie groups and dressing transformations, in "Integrability of nonlinear systems", Lecture Notes in Phys., 495, Springer, Berlin, (1997), 104-170.

[21] T. Ma and L. Liu, Rota-Baxter coalgebras and Rota-Baxter bialgebras, Linear Multilinear Algebra, 64(5) (2016), 968-979.

[22] A. Makhlouf and D. Yau, Rota-Baxter Hom-Lie-admissible algebras, Comm. Algebra, 42(3) (2014), 1231-1257.

[23] D. Manchon, Hoft algebras, from basics to applications to renormalization, Comptes-rendus des Rencontres mathematiques de Glanon, 2001.

[24] M. Marcolli and X. Ni, Rota-Baxter algebras, singular hypersurfaces, and renormalization on Kausz compactifications, J. Singul., 15 (2016), 80-117.

[25] J. Pei, C. Bai and L. Guo, Splitting of operads and Rota-Baxter operators on operads, Appl. Categ. Structures, 25(4) (2017), 505-538.

[26] G.-C. Rota, Baxter algebras and combinatorial identities I, II, Bull. Amer. Math. Soc., 75 (1969), 325-329, 330-334.

[27] M. A. Semenov-Tian-Shansky, What is a classical R-matrix? Functional Anal. Appl. 17(4) (1983), 259-272. 
[28] T. Zhang, X. Gao and L. Guo, Hopf algebras of rooted forests, cocyles, and free Rota-Baxter algebras, J. Math. Phys., 57(10) (2016), 101701 (16 pp).

[29] X. Zhang, X. Gao and L. Guo, Commutative modified Rota-Baxter algebras, shuffle products and Hopf algebras, Bull. Malays. Math. Sci. Soc., (2018), https://doi.org/10.1007/s40840-018-0648-3.

\section{Xigou Zhang}

College of Mathematics and Information Science

Jiangxi Normal University

Nanchang, Jiangxi 330022, China

e-mail: xyzhang@jxnu.edu.cn

\section{Xing Gao}

School of Mathematics and Statistics

Key Laboratory of Applied Mathematics and Complex Systems

Lanzhou University

Lanzhou, Gansu 730000, China

e-mail: gaoxing@lzu.edu.cn

Li Guo (Corresponding Author)

Department of Mathematics and Computer Science

Rutgers University - Newark

Newark, NJ 07102, USA

e-mail: liguo@rutgers.edu 